\title{
Monitoring yeast fermentations by nonlinear infrared technology and chemometrics- understanding process correlations and indirect predictions
}

\author{
Pontius, Katrin; Junicke, Helena; Gernaey, Krist V.; Bevilacqua, Marta
}

Published in:

Applied Microbiology and Biotechnology

Link to article, DOI:

$10.1007 / \mathrm{s} 00253-020-10604-0$

Publication date:

2020

Document Version

Peer reviewed version

Link back to DTU Orbit

Citation (APA):

Pontius, K., Junicke, H., Gernaey, K. V., \& Bevilacqua, M. (2020). Monitoring yeast fermentations by nonlinear infrared technology and chemometrics-understanding process correlations and indirect predictions. Applied Microbiology and Biotechnology, 104, 5315-5335. https://doi.org/10.1007/s00253-020-10604-0

\section{General rights}

Copyright and moral rights for the publications made accessible in the public portal are retained by the authors and/or other copyright owners and it is a condition of accessing publications that users recognise and abide by the legal requirements associated with these rights.

- Users may download and print one copy of any publication from the public portal for the purpose of private study or research.

- You may not further distribute the material or use it for any profit-making activity or commercial gain

- You may freely distribute the URL identifying the publication in the public portal 


\title{
Monitoring yeast fermentations by non- linear infrared technology and chemometrics - understanding process correlations and indirect predictions
}

$\underline{\text { Katrin Pontius }^{* 1}}$, Helena Junicke ${ }^{1}$, Krist V. Gernaey ${ }^{1}$ and Marta Bevilacqua ${ }^{2}$

*corresponding author: katrin.pontius@web.de

1: Technical University of Denmark, Department of Chemical and Biochemical Engineering, Process and Systems Engineering Center (PROSYS), Søltofts Plads, Building 228 A, 2800 Kgs. Lyngby, Denmark

2: University of Copenhagen, Department of Food Science, Ingredient and Dairy Technology, Rolighedsvej 26, 1958 Frederiksberg C, Denmark

\begin{abstract}
Fermentation processes are still compromised by a lack of monitoring strategies providing integrated process data online, ensuring process understanding, control and thus, optimal reactor efficiency. The crucial demand for on-line monitoring strategies, not only encouraged by the PAT initiative, but motivated by modern paradigms such as circular economy and sustainability, has driven research and industry to provide 'next generation process technology': in other words, technology tailored towards industrial needs. Mid-infrared (MIR) spectroscopy as such is superior to nearinfrared (NIR) spectroscopy since it provides significantly enhanced selectivity. However, due to high costs and a lack of instrumental robustness, MIR spectroscopy is outcompeted by NIR when it comes to industrial application. The lack of chemometric expertise, model understanding, and practical guidance might add to the slow acceptance of industrial MIR application. This work demonstrates the use of novel MIR, so-called non-linear infrared (NLIR) technology and the importance of model understanding, exemplarily investigated on a lab-scale yeast fermentation process. The six analytes glucose, ethanol, glycerol, acetate, ammonium and phosphate were modeled by partial-least-squares (PLS) based on spectral data, demonstrating the potential of the novel technology facilitating on-line data acquisition and the necessity of investigating indirect predictions.
\end{abstract}




\section{Key Points}

- $\quad$ NLIR spectra were acquired on-line during a yeast fermentation process

- $\quad$ PLS models were constructed for six components based on uncorrelated samples

- Glucose, ethanol, ammonium and phosphates were modeled with errors of less than $15 \%$

- Acetate and glycerol were shown to rely on indirect predictions

\section{Keywords}

Mid-infrared spectroscopy, PLS modeling, on-line monitoring, indirect predictions, yeast fermentation 


\section{Introduction}

Bioprocess manufacturing has played a key role in the food, pharma and the chemical industry for the last 50 years. The producing core of the biotech industry is the fermentation process itself, which is often considered as the most complex unit operation within bio-manufacturing. Modern industrial processing poses paradigms such as circular economy and sustainability to any industry. In this regard, monitoring and control strategies are the key requirements to set new standards, aiming at eliminating the environmental burden while increasing the productivity and running the process at optimal reactor efficiency. In contrast to this ideal, yet crucial for future bioprocessing, fermentation process operation is still generally challenged by a lack of tools for process monitoring and control. Control actions - frequently based on experience instead of process data - are still executed manually to a high degree, relying on the experience of the process operator. Outside the well-established and trusted sensor systems for $\mathrm{pH}$, temperature, dissolved oxygen, and in well-equipped laboratories and industries, off-gas analysis, real-time measurements of biomass, substrate and product concentrations are rare and generic solutions are highly challenged by medium and process complexity. The process analytical technology (PAT) framework published in 2004 (FDA 2004) has addressed this crucial demand of process understanding aiming at integrated control throughout appropriate monitoring strategies. Besides, a new driving force has been created by the vison of Industry 4.0 (Hermann et al. 2016) announced as one of the key initiatives at first in 2011 by the German federal government. The convergence of industrial production, process data and cloud communication can only be achieved on the basis of valid monitoring strategies. Consequently, both, academia and industry, have been increasingly focusing on providing generic monitoring solutions.

Within the PAT framework, the interaction of molecules with light has been widely used for their detection and quantification as exemplarily outlined by Pavia et al. (2001). Due to their fast, high-throughput, informative and nondestructive nature, vibrational spectroscopic techniques, in particular near $\left(4000-13000 \mathrm{~cm}^{-1}\right)$ and mid $(2000-$ $4000 \mathrm{~cm}^{-1}$ ) infrared spectroscopy (NIRS and MIRS, Larkin 2011; Sun 2009) combined with multivariate data analysis (Beebe and Kowalski 1987; Miller 2000; Steele 1990) have been shown to be highly powerful for achieving improved process understanding and control. Both, NIR and MIR spectroscopic techniques can be applied to the fermenter by means of fiber optical probes. It might be due to the more robust and flexible, yet less expensive design of a NIR spectroscopic set-up that NIR applications are more established in a production process environment than MIR spectroscopy. However, as demonstrated by Landgrebe et al. (2010), MIR spectroscopy clearly scores higher than NIRS when it comes to selectivity. Fundamental molecular vibrations are excited by the MIR region whereas the overtones 
and vibration combinations are excited by the NIR region. Fundamental vibrations cause a larger change in energy than the excitation of overtones and thus, MIR absorption spectra yield more distinctive bands than NIRS (Landgrebe et al. 2010). In other words, MIR spectra are more informative than NIR spectra. In a MIR spectrum, the so-called fingerprint region ranging from approximately 800 to $1800 \mathrm{~cm}^{-1}$ is especially descriptive. It responds to all functional groups (e.g. alkyl, alcohol, carboxyl, carbonyl, amide, phosphate) constituting organic components. Thus, MIR produces exquisite molecular selectivity and, compared to NIRS, its greater resolution enables the prediction of a single analyte at significantly lower concentration levels, especially in a complex culture medium as the fermentation broth. Consequently, MIRS and, in this field especially, Fourier transform infrared spectroscopy (FTIR) have become increasingly interesting for multi-component monitoring in bioprocesses. Its stagnancy in industrial biotech applications, however with incremental progress, might be due to the high cost, the presence of fragile and sensitive moving parts making it sensitive towards mechanical vibrations, intrinsic thermal noise and the limited length of attenuated total reflectance (ATR) fibers for remote sampling. These challenges are addressed by recent technical advances in the MIR sensor technology, as for instance described in Bogomolov et al. (2015) and besides, employed in this work (Barh et al. 2017; Dam et al. 2016). These novel technologies use simpler grating spectrometers, are free of any moving parts, reduce intrinsic detector noise and are significantly less expensive than traditional high-precision FTIR analyzers, typically employing the full spectral range $\left(800-4000 \mathrm{~cm}^{-1}\right)$. However, the full spectral range is rarely necessary in bioprocess monitoring as information about the main nutrients and products can be obtained from the fingerprint region. Thus, apart from increased robustness and affordability, novel technology tailors the spectrometer to this highly informative region. The versatility of MIRS in combination with multivariate calibration models such as partial-least-squares (PLS) has been demonstrated and reviewed in several publications as a powerful tool for monitoring of fermentation processes (Dahlbacka et al. 2012; Fayolle et al. 2000; Genkawa et al. 2012; Koch et al. 2014; Kornmann et al. 2004; Landgrebe et al. 2010; Lourenço et al. 2012; Pollard et al. 2001; Sakhamuri et al. 2001). While the potential of this combined approach is immense as referenced before, practice shows that the application is challenged by a lack of expertise, intensive calibration procedures and finally, often a lack of model robustness. The lack of model robustness might boil down to a lack of model understanding and lacking maintenance procedures. Multivariance is the strong advantage of IR spectroscopy and an intrinsic challenge. Especially in fermentation processes, most components of interest are IR active comprising the same functional groups. Hence, spectral covariance resulting from overlapping spectral features, together with highly correlated fermentation process dynamics (Petersen et al. 2010), are a common reason for indirect predictions. In regards of fermentation monitoring by IR spectroscopy and 
chemometric modeling, indirect predictions remain poorly addressed. This work presents the first report of predicting all the relevant yeast performance parameters glucose, ethanol, glycerol and acetate by the use of the novel NLIR technology combined with PLS modeling. Additionally, prediction models were built for ammonium and phosphates as regular nutrients in various fermentation processes, however not particularly important to the presented work, yet relevant to other processes as described by Pontius et al. (2019). The modeling of the six components based on NLIR technology exemplarily outlines the challenge of indirect predictions in the framework of the employed yeast process and points out the biological and spectral cage of covariance (Berhe et al. 2016; Eskildsen et al. 2014). It is important to realize that biological correlation and overlapping spectral features might be the main reason for indirect, non-robust models and that this information is not captured by the PLS model itself. The work presents important considerations when dealing with this powerful, yet highly complex strategy of IR spectroscopy and PLS modeling, evaluating model performance beyond the conventionally used parameters such as the coefficient of determination $\left(\mathrm{R}^{2}\right)$ and the rootmean-square-error of cross validation (RMSECV). Finally, the inclusion of novel NLIR technology strikes the technical progress towards monitoring in an industrial process environment by IR instrumentation.

\section{Material and Methods}

\section{Data sets}

Six data sets were prepared for PLS model calibration and validation comprising a total of 137 samples analyzed in duplicate by IR spectroscopy with two different instruments (novel NLIR and standard FTIR) and reference methods. For this purpose, the spectral data was collected off-line. It included the data of four fermentation batches, a set of synthetic samples in order to decouple the process correlations and a set of spiked (semi-synthetic) samples to evaluate model robustness (Petersen et al. 2010). Additionally, spectral data was collected on-line by exchanging the ATR unit of the NLIR instrument with an ATR unit designed as a flow-through-cell. An overview of the seven data sets is presented in Table 1. 
Table 1: Data set overview; $C E N P K$ refers to the yeast strain $C E N P K$ 113-7D, SIC refers to the insulin precursor (IP) producing yeast strain as described in Liu et al. (2012)

\begin{tabular}{lll}
\hline Data set Nr. & Type / Nr. of samples & Description \\
\hline $\mathbf{1}$ & CENPK / 20 & $\begin{array}{l}\text { Batch fermentation; off-line spectral analysis of cell-free } \\
\text { supernatant; }\end{array}$ \\
\hline
\end{tabular}

$2 \quad$ CENPK / 19

$3 \quad$ SIC / 19

$4 \quad$ CENPK / $16(\mathrm{x} 2)$

$5 \quad$ CENPK / $16(\mathrm{x} 2)$

$6 \quad$ Synthetic samples / 15
Fed-batch fermentation; spiked with ammonium (at time 0), phosphate and glucose (after $6 \mathrm{~h}$ ), off-line spectral analysis of cell-free supernatant;

Batch fermentation, IP producing yeast, off-line spectral analysis of cell-free supernatant;

Batch fermentation, off-line spectral analysis of both cell-free supernatant and cell- containing samples;

Spiked samples obtained from data set 4, off-line spectral analysis of both cell-free supernatant and cell-containing samples;

Synthetic samples containing all the six components of interest in unnatural proportions designed to minimize the correlations between the analytes;

CENPK / 150 on-line

Fed-batch fermentation; spiked with glucose after 9 h; Spectral data was collected on-line over 25 hours. Every $6^{\text {th }}$ on-line spectrum was complemented with reference data obtained from a manual withdrawn sample.

\section{Yeast fermentation processes}

Yeast fermentations were performed by cultivating the yeast strain $C E N P K-1137 D$ (available on request from the EUROSCARF collection, Germany), used widely in fundamental and applied research, and, respectively, the industrially relevant insulin precursor (IP) producing yeast strain (referenced as SIC and described by Liu et al. (2012), available on request from Chalmers University, Sweden). Both yeast strains were grown for 24-30 h in complex (YPD) medium, in a working volume of $2 \mathrm{~L}$, at $\mathrm{pH} 6$, a temperature of $30{ }^{\circ} \mathrm{C}$, an agitation speed of $800 \mathrm{rpm}$ and an aeration rate of 1 vvm. $\mathrm{pH}$ control was achieved by adding $2 \mathrm{M} \mathrm{H}_{2} \mathrm{SO}_{4}$ and $2 \mathrm{M} \mathrm{KOH}$ as $\mathrm{pH}$ reagents. The YPD medium contained per liter of water: $10 \mathrm{~g}$ yeast extract (' $\mathrm{Y}$ ', Merck, Courbevoie Cedex, France), $20 \mathrm{~g}$ peptone ('P', Merck, Mexico City, Mexico) and 20 g glucose (dextrose, 'D', Center Valley, Macron, USA). The dissolved oxygen tension stayed above $30 \%$ of saturation across all fermentations performed, indicating that no oxygen limitations occurred during the processes. The fermenter equipment and eZ controller were purchased from Applikon, Delft, The Netherlands. For reference and spectral analysis, samples were withdrawn manually and filtered via a $0.2 \mu \mathrm{m}$ sterile 
filter (Sartorius, Göttingen, Germany). Glucose, ethanol, glycerol and acetate were analyzed by high-performance liquid chromatography (HPLC), while phosphates and ammonium were analyzed by specific assay kits as described below in Reference analysis.

\section{Design and preparation of synthetic and spiked samples}

\section{Design of synthetic samples}

The fermentation parameters analyzed are inherently trapped in a biological cage of covariance, meaning that they are highly correlated with each other due to the microbial metabolic network activity they undergo. A set of 15 synthetic samples was included in the calibration data (data set 6 in Table 1), containing the six analytes of interest in artificial proportions. Inclusion of these uncorrelated samples served to decouple the process correlations, supporting the PLS model to find unique regression vectors based on spectral information for each of the components. The reference matrix for the synthetic samples was a first yeast batch fermentation, described by the concentration values of the six analytes over a time span of $16 \mathrm{~h}$ (15 samples), with reference values obtained hourly over the batch phase. Hence, the reference data matrix was spanned by 15 rows (concentration values for every hour) and 6 columns (representing the six components). The fermentation profile of the reference yeast batch process in relation to data sets $1-7$ is shown in Supplementary Fig. S1. Generally, 15 process values of each component yielded a comprehensive fermentation profile for this process, representing entirely the concentration range required for each component to be modelled. Hence, 15 synthetic samples were prepared, appropriate to decouple the process correlations. Ammonium and phosphates were added up to $100 \mathrm{mM}$ in order to incorporate a greater variability relevant to other processes, as the inorganic ions as such were only present in very low amounts in the complex medium used for fermentation. The design matrix for the synthetic samples was obtained by permutation of the row values individually for each component, aiming at minimizing the correlation between them. For practical reasons, the concentration values were rounded to the nearest 5 mM. Hence, the design matrix for the synthetic samples represented realistic process values, but in unrealistic proportions.

\section{Preparation of synthetic and spiked samples}

The synthetic samples (data set 6 in Table 1) were prepared by adding the right amount of a 2 M stock solution of each component to the glucose - free (blank) fermentation medium ('YP' medium) according to the design matrix defined before, and adjusted to the operational $\mathrm{pH}$ of 6. 
In order to validate the model robustness, samples from data set 4 were spiked. The prediction of the spiked samples will be accurate if the model is built upon direct correlation based on distinct spectral features connected to the analyte of interest. If the prediction of the spiked samples is biased, it will hint towards indirect predictions of this component. In other words, in such a case the model predicts the component based on a correlation by chance to spectral regions that are not connected to the actual spectral fingerprint of that component. The spiking was applied to the fermentation samples and hence, no 'clean' background was present as was the case for the synthetic samples. To keep the spiked samples approximately inside the concentration space spanned by the reference data matrix (see Design of synthetic samples), the reference data matrix was subtracted from the design matrix obtained for the synthetic samples. If the reference concentration value was higher than the concentration value aimed for in the synthetic (spiked) sample, the value in the spiking scheme was set to zero and nothing was added for the respective component. Otherwise, the difference between reference data point and designed data point was added to obtain the actual design point for the spiked sample. Also here, ammonium and phosphates were added up to $100 \mathrm{mM}$ in order to incorporate a greater variability relevant to other processes. As for the synthetic samples, the final concentration value was rounded to the nearest $5 \mathrm{mM}$. Spiking was performed right after sampling by adding the appropriate amount of a $2 \mathrm{M}$ stock solution.

Stock solutions for both, synthetic and spiked samples, were prepared with a final concentration of $2 \mathrm{M}$ and adjusted to pH 6 as follows: Glucose, acetate and ammonium stock solutions were prepared by dissolving the appropriate amounts of solids (glucose ((dextrose), Macron, Center Valley, USA), sodium acetate trihydrate (Sigma, St. Louis, USA) and ammonium chloride (Sigma, St. Louis, USA), respectively) in purified water. Stock solutions of phosphates, glycerol and ethanol were prepared by adding the appropriate amount of $85 \%$ phosphoric acid (Aldrich, Nantong, China), glycerol (VWR chemicals, Heverlee, Belgium), and ethanol (Sigma Aldrich, Munich, Germany), respectively, to purified water. All solutions were adjusted to $\mathrm{pH} 6$ by the addition of a few drops of $\mathrm{NaOH}$ or HCL, respectively.

\section{Pure component solutions for the investigation of pure component spectra}

Solutions of the pure components were prepared in purified water in order to investigate their spectral fingerprint. Each component was prepared in solutions with a concentration of 10,50,100 and $200 \mathrm{mM}$ in order to identify the related peaks in the spectra. The solutions were adjusted to pH 6 by adding a few drops of $\mathrm{NaOH}$ (Merck, Darmstadt, Germany) or HCL (Thermo Fisher Scientific, Bremen, Germany), respectively. Note that, the $\mathrm{pH}$ reagents used for $\mathrm{pH}$ adjustment were not IR active. 


\section{Reference analysis}

All samples were analyzed in duplicate. Glucose, glycerol, acetate and ethanol were quantified by HPLC equipped with refractive index detector via the Aminex HPX $87 \mathrm{H}$ column with a standard deviation of below $3 \%$ across all samples measured. Colorimetric assay analysis was performed to quantify ammonium and phosphates with a standard deviation below $5 \%$. Detailed description of the reference methods can be found in Supplementary Material S1.

\section{MIR analysis}

IR spectra were acquired in duplicates on two different instruments. One instrument can be considered as a commercial standard lab-instrument (ABB Bomem MB100, ABB Bomem Inc., Québec, Canada) based on the ATR-FTIR principle. The other instrument was the novel NLIR technology described below. The NLIR instrument used was a prototype in development kindly provided by NLIR Aps (Nonlinear Infrared Sensors, Farum, Denmark). PLS models were developed based on spectral data acquired with both instruments in order to compare the quality of the spectral data.

\section{ATR-FTIR (standard instrument)}

Infrared spectra were acquired off-line on the FT-spectrometer ABB Bomem MB100, equipped with a Globar silicon Carbide ( $\mathrm{SiC}$ ) radiation source collimated to a $2.5 \mathrm{~cm}$ diameter beam and a deuterated triglycine sulfate (DTGS) detector. The measurements were performed in ATR mode, by putting $800 \mu \mathrm{L}$ of each sample on the ATR cell (ZnSe crystal, $45^{\circ} \mathrm{C}$ ) and recording a full spectrum between 750 and $4000 \mathrm{~cm}^{-1}$ with 64 scans at a resolution of $8 \mathrm{~cm}^{-1}$. The background was obtained on an empty ATR groove against air using 128 scans. In between the samples, the ATR groove was cleaned with water and $70 \%$ ethanol and dried out with spectrophotometric tissues. Subsequently, only the region between 900 and $1550 \mathrm{~cm}^{-1}$ was kept for further analysis. This region refers to the region facilitated by the NLIR instrument. Besides, it is defined as the so-called fingerprint region, which is rich in spectral information linked to the different functional groups present in biological samples.

\section{NLIR - Nonlinear IR (prototype)}

The NLIR instrument is based on novel technology, featured in detail by Barh et al. (2017), Buse et al. (2017) and Dam et al. (2016). Unlike the conventional FTIR spectrometer as used above, in which the most important part is represented by the interferometer, as described by Subramanian and Rodriguez-Saona (2009), the core technology in the NLIR instrument is a $\mathrm{AgGaS}_{2}$ crystal inside a laser cavity. The IR light passes through the ATR and enters the crystal inside the laser cavity in which a process called upconversion takes place. The upconversion process converts the incoming IR 
light that has interacted with the sample to near-visible light. The spectral information in the upconverted light is completely conserved. A standard silicon-based visible-light detector (CCD) was used for detection, which is efficient, free from intrinsic thermal background noise (compared to a conventional IR detector that emits IR light itself due to its own temperature (black body radiation), thus, adding noise) and cheap. Besides, visible-light detectors are orders of magnitudes better in terms of detectivity than MIR light detectors, clearly highlighting the advantage of the upconversion process. The combination of upconversion and visible-light detector is the key to fast and efficient MIR wavelength detection in the NLIR spectrometer. Furthermore, the optical core of this technology does not contain any moving parts (a moving mirror is typically part of an interferometer) resulting in increased robustness against mechanical vibrations. The term nonlinear (NL) IR results from the field of nonlinear optics to which the theoretical background of the upconversion process belongs.

It must be highlighted that spectroscopic studies with this instrument have rarely been conducted on fermentation samples and that the case presented here is the first to be published within the bioprocess field.

\section{Off-line measurements}

The NLIR instrument was equipped with a $1200 \mathrm{~K}$ silicon nitride filament as infrared light source and a grating spectrometer with a silicon-based CCD line array as detector. The measurements were performed in ATR mode, by putting $800 \mu \mathrm{L}$ of sample on the ATR cell (ZnSe crystal, $45^{\circ} \mathrm{C}, 12$ bounces) and recording a spectrum between 800 and $1800 \mathrm{~cm}^{-1}$ with 100 scans at a resolution of $10 \mathrm{~cm}^{-1}$ and an exposure time of $40 \mathrm{~ms}$. The background was obtained on an empty ATR groove against air using 100 scans. In between the samples, the ATR groove was cleaned with water and $70 \%$ ethanol and dried out with spectrophotometric tissues. Subsequently, only the non-noisy region, between 900 and $1550 \mathrm{~cm}^{-1}$, was kept for further analysis.

\section{On-line measurements}

Aiming at developing an on-line set-up for bioprocess monitoring by IR spectroscopy, mid-IR measurements with the NLIR instrument were conducted on-line during a final yeast fed-batch fermentation (data set 7 in Table 1). The stationary ATR (ZnSe crystal, $45^{\circ} \mathrm{C}, 12$ bounces) was exchanged with an ATR crystal $\left(\mathrm{ZnSe}\right.$ crystal, $45^{\circ} \mathrm{C}, 10$ bounces) designed as a flow through-cell (HATR Flow-Through Cell, PIKE Technologies, USA). The fermentation broth was recirculated from the standard sampling port inside the reactor to the ATR flow-through-cell, and via a pump (Ismatec Reglo ICC, Ismatec, Germany) circulated back to a separate fermenter inlet. The sampling port was equipped 
with a $20 \mu \mathrm{m}$ stainless steel filter cap (sample screen $20 \mu \mathrm{m}$, Applikon, The Netherlands) to prevent the sampling of air bubbles. The recirculation loop was operated with a flow rate of $5 \mathrm{ml} / \mathrm{min}$ facilitating an exchange of fermentation broth inside the flow-through-cell every minute. IR spectra were automatically recorded every 10 minutes over $25 \mathrm{~h}$, while a manual sample for reference analysis was taken every hour via a second sampling port. Thus, every $6^{\text {th }}$ IR measurement was complemented with off-line reference data of the components of interest.

\section{Chemometric analysis}

Chemometric analysis was performed in MatLab R2017b (MathWorks, Natick, Massachusetts), using both the PLS_Toolbox (Eigenvector Research Inc., Manson, Washington) and in-house written routines. Briefly, The PLS algorithm describes the relationship (calibration model) between the analyte concentrations (y) and the IR spectrum (data matrix $\mathbf{X}$ ). It is suitable for handling the regression of $\mathbf{y}$ on numerous, highly correlated variables as presented by IR spectra. The PLS model approximates the spectral data by a smaller number of so-called latent variables. The latent variables are linear combinations of the original spectral values describing the variation in $\mathbf{X}$ covarying with the information in $\mathbf{y}$. They are obtained by maximizing the covariance between $\mathbf{X}$ and $\mathbf{y}$ using a least square approach. The calibration yields a PLS model which can be used for the prediction of analyte concentration based on spectral data only.

PLS calibration models as described in Geladi and Kowalski (1986) and Sjöström et al. (1983), were built using the SIMPLS algorithm and a 10-fold cross-validation procedure that kept all spectral replicates in the same subset (Westad and Marini 2015). The spectral raw data of the calibration set was subject to mean-centering and the first derivative was taken using the Savitzky-Golay algorithm (Savitzky and Golay 1964) (window-size of 25 data points and a $3^{\text {rd }}$ order polynomial) as spectral preprocessing. Preprocessing with Savitzky-Golay, applying the first derivative and smoothing the spectra, yielded better models than spectral preprocessing with standard normal variate (SNV, Barnes et al. 1989), and second derivative and smoothing (Rinnan et al. 2009). The best preprocessing method, and in general, the model performance, was evaluated based on the model error (root-mean-square error of prediction, RMSEP) obtained as calculated in equation 1 according to the statistical calculations reported in Skou et al. (2017):

$\operatorname{RMSEP}[\%]=\sqrt{\frac{\sum_{i=1}^{N}\left(y_{i}-\hat{y}_{i}\right)^{2}[\mathrm{mM}]^{2}}{N}} \cdot \frac{1}{\Delta y_{v a l}[\mathrm{mM}]} \cdot 100$ equation 1

$\mathrm{N}$ equals the total number of samples in the validation set, $\mathrm{y}_{\mathrm{i}}$ equals the reference value of sample $\mathrm{i}, \hat{y}_{\mathrm{i}}$ equals the predicted value of sample $\mathrm{i}$ and $\Delta y_{v a l}$ equals the concentration range present in the validation set. RMSEP values were 
calculated based on external validation. As such, the data used for model validation was not included in the calibration set and belonged to a separated fermentation batch. Generally, this yields a more realistic idea of the model performance compared to the commonly used root-mean-square-error of-cross-validation (RMSECV, Westad et. al. 2013). However, the RMSECV often results in an overoptimistic idea of the model performance as it does not account for batch-to-batch variability and indirect predictions. The models were furthermore assessed by means of the variable importance in prediction (VIP, Wold et al. 1993, implemented in the PLS toolbox) index (scores). A VIP index (VIP scores) greater than 1 generally indicate a high relevance of the respective variables (spectral region) for the PLS model.

Principle Component Analysis (PCA) (Pearson 1901; Wold et al. 1987) was performed as an explorative method to describe and classify the spectral variance observed in data sets $1-7$. In PCA, $\mathbf{X}$ is approximated by latent variables as in PLS. However, the model is derived from the spectral data without considering the response variables (y) and thus, the latent variables represent the 'natural' classification of the spectra. The PCA model was built on the whole spectral data of samples (date set $1-7$, Table 1). The spectra were mean centered and subjected to the first derivative pretreatment, aiming at a PCA model describing the spectral variance on the preprocessed data as used for the PLS calibration. 


\section{Results}

\section{Process analysis}

The seven data sets presented in Table 1, are shown per component in Fig. 1.
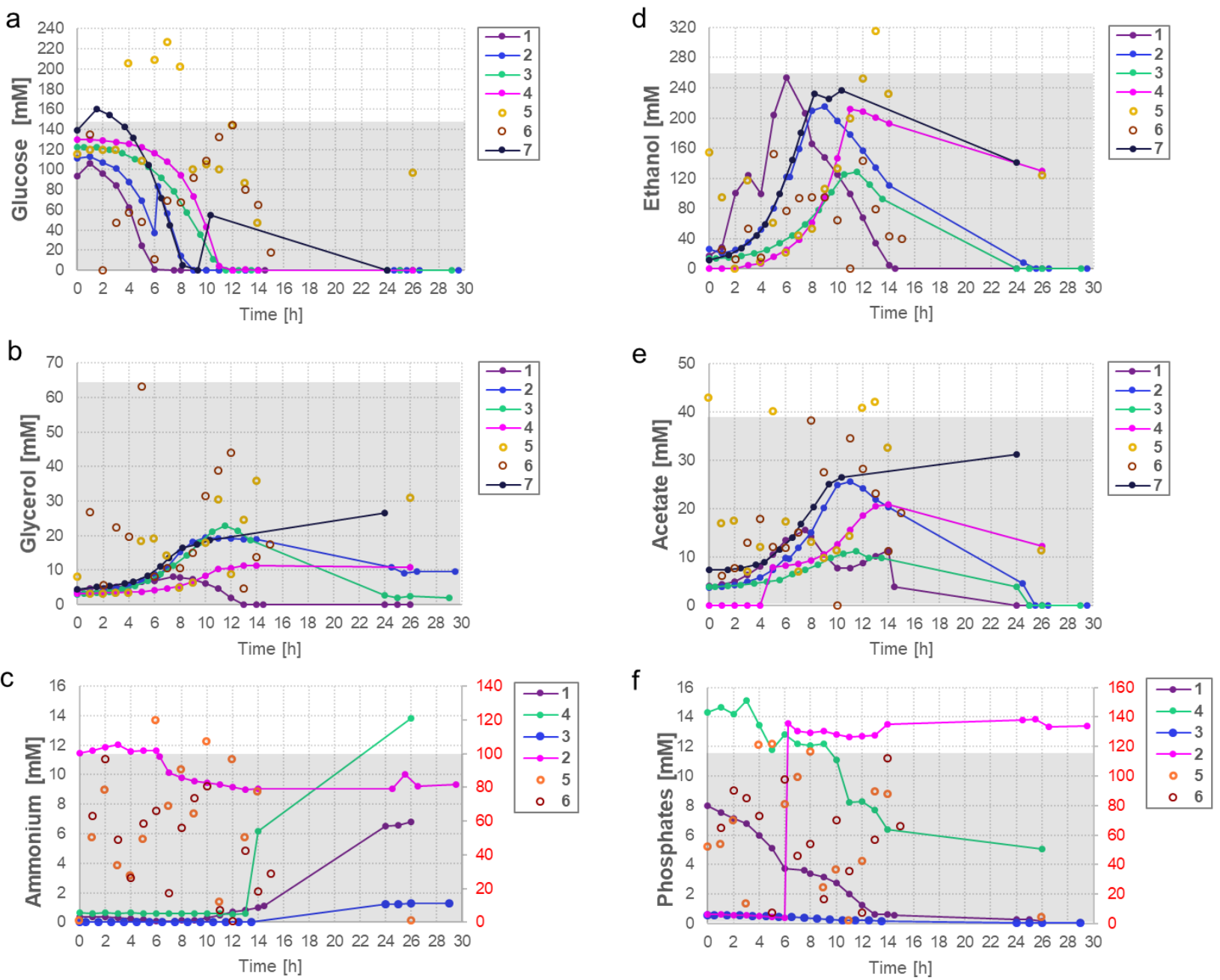

Fig.1: Concentration profiles per component of the data set 1 - 7 as described in Table 1, determined by HPLC and assay analysis. Note, that each data point represents the average of two measurements (standard deviation $<3 \%$ ). The solid lines connecting the data points within the fermentation data account for easier visualization and not for continuous data. The grey area marks the space spanned by data set 1 and 6 , used for PLS model calibration. In case of data set 6, the $x$-axis refers to the sample number, as no time trend is implied in these samples. a: Glucose-. b: Glycerol-. c: Ammonium-. d: Ethanol-. e: Acetate-. f: Phosphates-profiles. In case of the data for ammonium and phosphates, the right-hand side y-axis refers to sample set 2, 5 and 6 (spiked and synthetic samples, addition of and ammonium), and the left-hand side y-axis refers to data set 1, 3 and 4 (fermentation batches excluding the addition of phosphate and ammonium) 
Fig. 1 was evaluated from both, a physiological and a modeling perspective. From a physiological point of view, data sets $1-4$ and 7 assemble typical yeast fermentation processes. Glucose is consumed and mainly transformed into ethanol and biomass (biomass data not shown). Next to ethanol, acetate and glycerol are produced and secreted into the medium due to overflow metabolism. When the glucose is depleted, the diauxic shift is induced, and the cells start to grow on ethanol as well as on acetate and glycerol as secondary carbon and energy source. Detailed information about the yeast metabolism can be found in Sonnleitner and Käppeli (1986) and von Schalien et al. (1995). From a modeling point of view, the process data was subject to a rather high batch-to-batch variability. Besides, it was obvious that the variability space (concentration range) of glucose and ethanol (disregarding ammonium and phosphates in the spiked batches and synthetic samples, data sets 2, 5 and 6) was much higher than the variability space of the analytes acetate and glycerol. Not considering the spiked and synthetic samples, glucose and ethanol varied in a range of $0-144 \mathrm{mM}$ and $0-253 \mathrm{mM}$, whereas glycerol and acetate varied only up to $36 \mathrm{mM}$ and $43 \mathrm{mM}$, respectively. The levels of ammonium and phosphates in the YPD medium were found to be very low, around maximal $1 \mathrm{mM}$ and $15 \mathrm{mM}$. The concentration change of these two components must be considered as insufficient for PLS modeling within this process when not added to the medium in higher amounts and only the synthetic and spiked samples did account for reasonable variability of up to approximately $100 \mathrm{mM}$.

\section{Decoupling of process correlations}

Data sets 1 and 6 were used for PLS model calibration as accounting best for the overall variability across all data sets (data set 1 spanned the lower, data set 6 the upper variability observed across the data sets). The process correlations and their decoupling within the calibration data is presented in Fig. 2. 

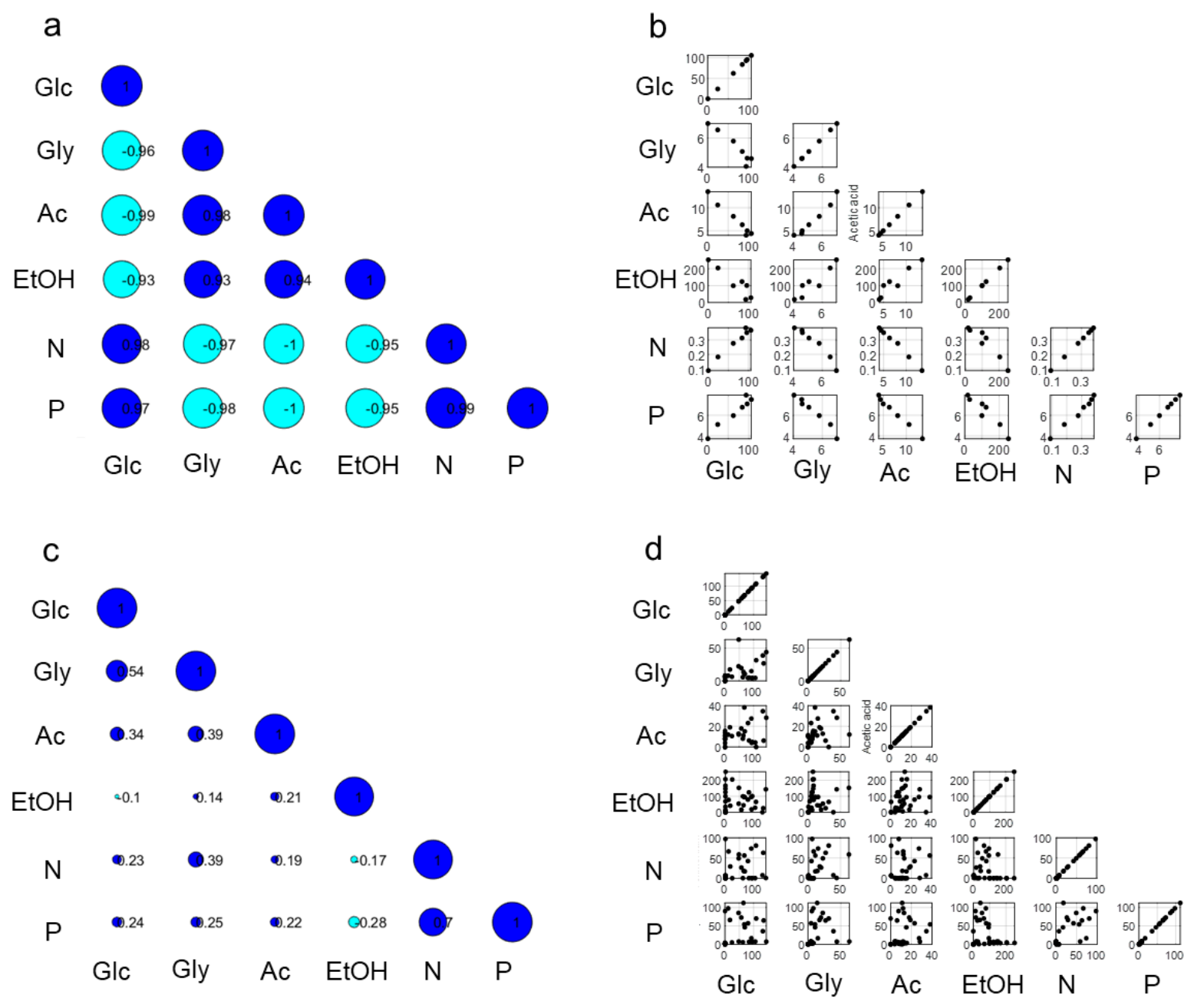

Fig.2: a: Bubble plot of the correlation coefficients ( $r$ values) within data set 1 (Glucose (Glc), Glycerin (Gly), Acetate (Ac), Ethanol (EtOH), Ammonium (N) and Phosphates (P)), corresponding to the initial batch-phase only (ending after $6 \mathrm{~h}$ with glucose depletion). In data set 1 , the average correlation between two components is very strong with $r=0.968$ on average. $b$ : The scatter plots behind the $r$ values presented in a. c: Bubble plot of the correlation coefficients within the combined data set 1 and 6. The average correlation between two components is very weak with $r=0.293$ on average. The correlations in data set 1 were decoupled by the inclusion of the synthetic samples (data set 6 ). d: The scatter plots according to the $r$ values presented in c. The bubble plots represent values from -1 to 1 . The dark blue color indicates a positive correlation, the light blue color indicates a negative correlation between two components. The bubble size correlates to the strength of the correlation. Respectively, a large bubble ( $r$ close to 1 or -1 ) represents a strong correlation, and a small bubble ( $r$ close to 0 ) represents a weak correlation between two components. The diagonal can be seen as a reference for the bubble size corresponding to an $r$ value equal to 1 (100 \% correlated)

As demonstrated in Fig. 2 a and b, the correlations between the components in the fermentation process are very strong, with an average correlation coefficient (Pearson correlation coefficient, r-value) of 0.968 . This holds especially for the exponential growth or the initial batch-phase. Including the process values after the diauxic shift lowered the average 
correlation between two components to an r-value of 0.506 . However, the correlations in the second growth phase as such, in which ethanol and in parallel, acetate and glycerol, are consumed, are comparably strong as in the first growthphase in which the yeast grows on glucose. Including the synthetic samples into data set 1 reduced the average correlation between two components to an r-value of 0.239 (Fig. $2 \mathrm{c}$ and d). With that, the process correlation could be considered as decoupled. As obvious in the scatter plot shown in Fig. 2 d, the concentration values of the different components vary now independently from each other, supporting the PLS model to find unique regression vectors for each component. It must be mentioned, that 15 (or more) samples equally spaced between the minimum and maximum value found for each component provided an improved design matrix for subsequent row value permutation. Uncorrelated samples resulting from such a design matrix were investigated outside this work and showed an even lower $\mathrm{r}$ and besides, a decreased leverage value, increasing the predictability in each data point.

\section{Spectral covariance - inspection of the pure component spectra}

IR radiation is absorbed by exciting fundamental vibrations of molecular bonds expressing a change in dipole moment. Thus, highly polar bonds result in absorption bands of higher intensity compared to less polar bonds. Generally, organic components are to a large extent composed of the atoms $\mathrm{C}, \mathrm{H}, \mathrm{O}, \mathrm{N}$ and $\mathrm{P}$, as the analytes examined in this work. Due to the difference in electronegativity of these atoms, bonds between them are inherently polar and prone to IR absorption. Stretching vibrations involving the relevant atoms C, H, O, N and P characteristically occupy the, so-called, fingerprint region of mid-IR spectra typically ranging from 800 to $1800 \mathrm{~cm}^{-1}$. The pure component spectra of all six analytes were investigated and their spectral fingerprint was assigned by the main peaks visible in the spectrum. The spectral region examined was reduced to $900-1550 \mathrm{~cm}^{-1}$ in order to exclude the strong water absorption band at 1630 $\mathrm{cm}^{-1}$. Pure component spectra of the six analytes glucose, ethanol, glycerol, acetate, ammonium and phosphates were acquired with the standard FTIR instrument, as well as with the NLIR instrument with both, the off-line and the flowthrough ATR element. Table 2 summarizes the peaks identified based on Supplementary Fig.s S2 - S4 and compares the findings to the peaks reported in the literature. 
Table 2: Peaks identified for each pure component in the fingerprint region $\left(900-1550 \mathrm{~cm}^{-1}\right)$, based on Supplementary Fig.s S2-S4. The absorption bands found were compared with literature and the excited vibrational mode was assigned

\begin{tabular}{|c|c|c|}
\hline component & $\begin{array}{c}\text { Peaks identified }\left[\mathrm{cm}^{-1}\right] \\
\text { FTIR / off-line ATR / flow-through ATR }\end{array}$ & $\begin{array}{lll}\text { excited vibration, literature } \\
\text { reference }\end{array}$ \\
\hline Glucose & $\begin{array}{l}1034 / 1043 / 1037 \\
1080 / 1080 / 1078 \\
1105 / 1112 / 1105 \\
1151 / 1158 / 1154\end{array}$ & $\begin{array}{l}\text { C-O stretch } \\
\text { C-O stretch } \\
\text { C-O stretch } \\
\text { C-O-C stretch } \\
\text { (Kornmann et al. 2003) } \\
\end{array}$ \\
\hline Glycerol & $\begin{array}{l}1041 / 1042 / 1040 \\
1111 / 1118 / 1106\end{array}$ & $\begin{array}{l}\text { C-O stretch } \\
\text { C-O stretch } \\
\text { (Sandor et al. 2013) }\end{array}$ \\
\hline Ethanol & $\begin{array}{l}1045 / 1047 / 1046 \\
1078 / 1090 / 1087\end{array}$ & $\begin{array}{l}\text { C-O stretch } \\
\text { C-O stretch } \\
\text { (Bogomolov et al. 2015; Kornmann } \\
\text { et al. 2003; Sandor et al. 2013) }\end{array}$ \\
\hline Acetate & $\begin{array}{c}-/-/ 1123 \\
1277 /-/- \\
1414 / 1420 / 1415\end{array}$ & $\begin{array}{l}\mathrm{C}-\mathrm{O}_{2} \text { symmetrical stretching } \\
\text { (carboxyl gr.) } \\
\text { C-O } \mathrm{O}_{2} \text { symmetrical stretching } \\
\text { (carboxyl gr.) } \\
\text { (Kornmann et al. 2003; Wu et al. } \\
2015 \text { ) }\end{array}$ \\
\hline Ammonium & $1456 / 1465 / 1454$ & $\begin{array}{l}\text { triply degenerated bending mode of } \\
\text { the tetrahedral ammonium ion } \\
\text { (Max \& Chapados 2013; } \\
\text { Roychoudhury et al. 2006; Schenk } \\
\text { et al. 2007; Wagner \& Hornig } \\
\text { 1950) }\end{array}$ \\
\hline Phosphates & $\begin{array}{c}\text { - / / } 992 \\
1076 \text { / } 1055 \text { / } 1076 \\
- \text { / } 1121 /- \\
1157 \text { / } 1156 \text { / } 1151\end{array}$ & $\begin{array}{l}\text { P-OH stretch } \\
\text { P-O2 stretch } \\
\text { (Klähn et al. 2004; Rudolph 2010) }\end{array}$ \\
\hline
\end{tabular}

Complementary, Fig. 3 shows exemplarily the pure component spectra collected with the FT-IR instrument (as considered as standard) at a concentration of $200 \mathrm{mM}$ in an overlaid fashion.

The predominant functional group present in glucose, ethanol and glycerol is the hydroxyl group. Consequently, these molecules excite absorption bands due to $\mathrm{C}-\mathrm{O}(\mathrm{H})$ stretching vibrations of the alcoholic groups which are located in the same spectral region, namely between 1000 and $1200 \mathrm{~cm}^{-1}$. The carboxyl group of acetate, as well as ammonium and phosphate as such can be seen as individual moieties causing a different spectral variation each. They cause absorption bands due to the $\mathrm{CO}_{2-}$ symmetrical stretching mode of the carboxyl group and furthermore N-H and P-O stretching 
vibrations excited in the ammonium- and phosphate- ion, respectively. The spectral variation associated with the phosphate ion occupies the spectral region between 1050 and $1300 \mathrm{~cm}^{-1}$, whereas the spectral fingerprint of ammonium and acetate occupies the spectral region from 1350 to $1500 \mathrm{~cm}^{-1}$. As evident in Fig. 3, spectral features were highly overlapping within this study and cannot be calibrated selectively by univariate regression. Glucose and phosphates show the highest molecular absorbance and dominate the region $1000 \mathrm{~cm}^{-1}-1300 \mathrm{~cm}^{-1}$. Hence, PLS modeling of ethanol and glycerol occupying the same spectral region but exhibiting significantly lower molecular absorbance might be prone to indirect correlation with the dominating species phosphates and glucose.

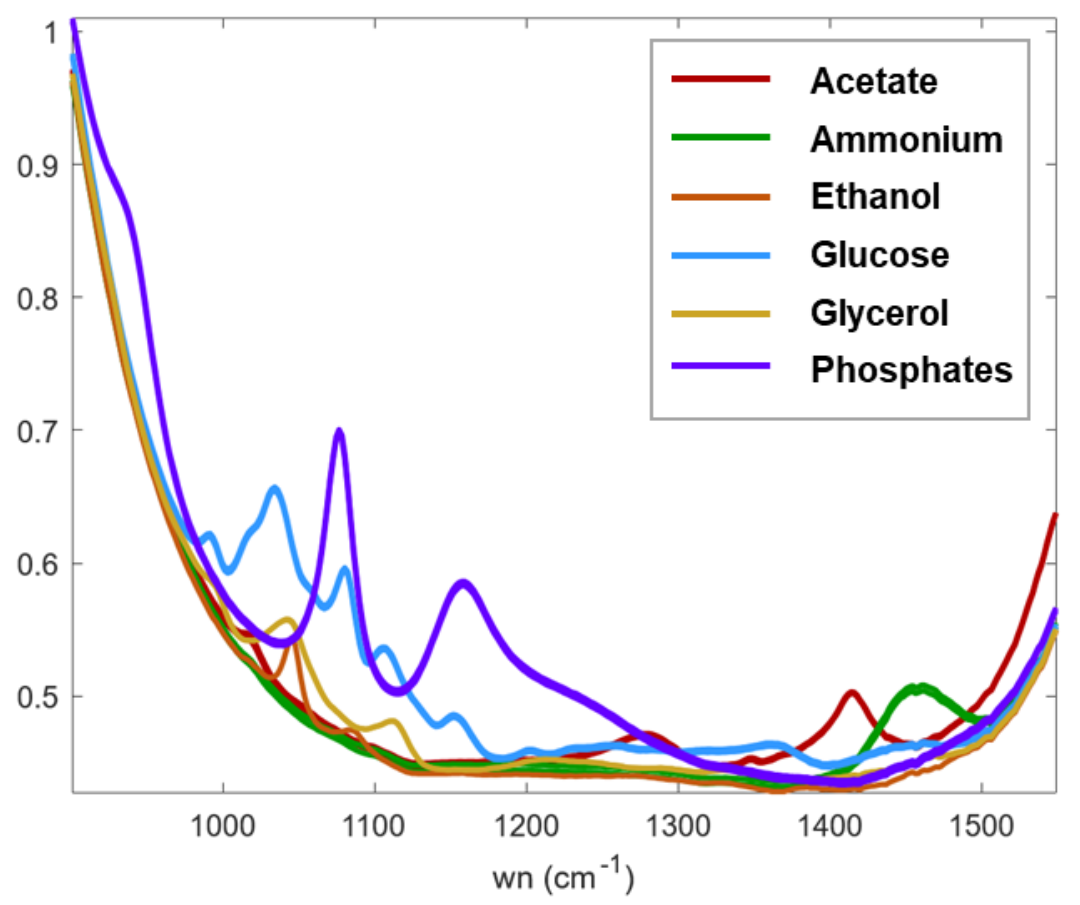

Fig.3: Pure component spectra collected with the FTIR instrument of a $200 \mathrm{mM}$ solution, in an overlaid fashion

In order to get an integrated picture of the IR absorbance inside the fermentation broth, the medium itself, the pH reagents for $\mathrm{pH}$ control, and the cells had to be considered. However, for the later evaluation of the PLS models, only the yeast cells and peptides in the fermentation medium should be kept in mind, described to cause a minor spectral variation at $1239 \mathrm{~cm}^{-1}$ (Sivakesava et al. 2001) and between $1200-1350 \mathrm{~cm}^{-1}$ (Singh 2009), respectively. A detailed discussion about the possible interferences arising from $\mathrm{pH}$ reagents, cells and peptides can be found in Supplementary Material S2. 
To demonstrate the performance of the NLIR technology, the focus will be on the NLIR data for the following results and discussion. The spectral and chemometric considerations presented can be considered as general conclusions in the field. Fig. 4 exemplarily shows the spectral evolution of data set 4 (cell-free samples, off-line spectra acquisition, Fig. 4 a) and data set 7 (cell-containing samples, on-line data acquisition, Fig. 4 b).

a

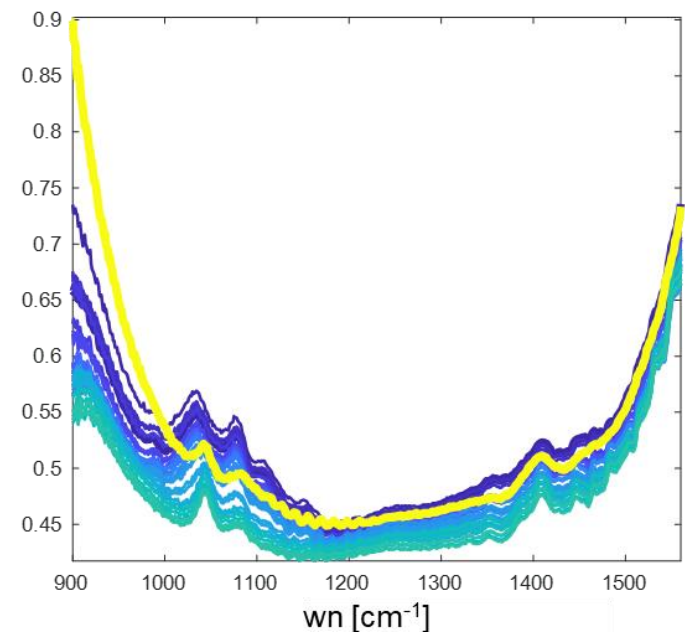

b

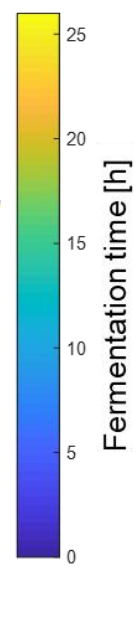

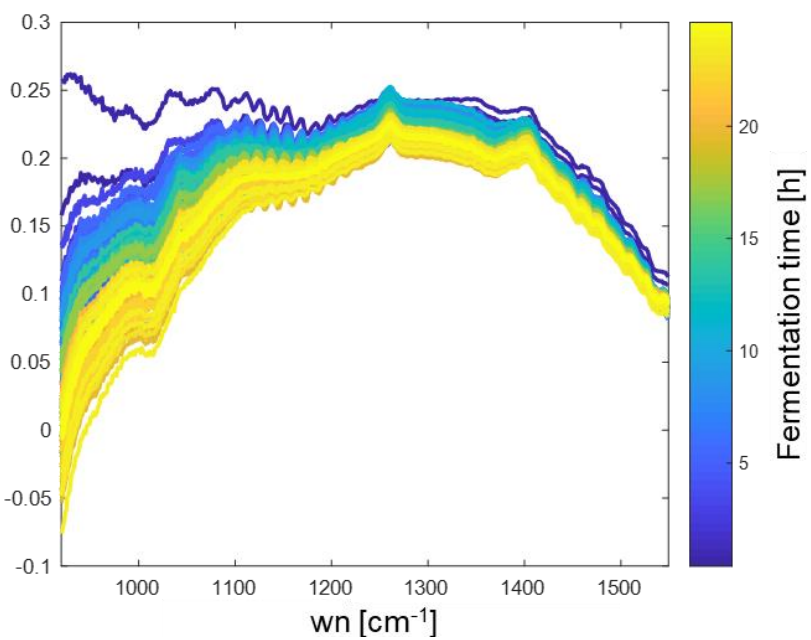

Fig.4: a: Spectral evolution over the fermentation course exemplarily demonstrated on data set 4 , for cell-free samples. The spectra were acquired off-line with the NLIR instrument at each data point as shown in Fig. 1. b: Spectral evolution over the fermentation course exemplarily demonstrated on data set 7 , for cell-containing samples. The spectral data was acquired online every 10 minutes at a flow rate of $5 \mathrm{ml} / \mathrm{min}$ with the NLIR instrument equipped with the ATR flow-through-cell

In both data sets, a clear spectral gradient can be observed according to the fermentation course, defined by metabolite production during the first $13 \mathrm{~h}$ followed by metabolite consumption after the diauxic shift. Note that, the base line shapes for off-line and on-line spectra were significantly different and this difference could be linked to the two different ATR-units in use, differing in surface area, surface shape and the number of bounces the light undergoes inside the ATR crystal. Besides, the application of flow slightly increased the noise in the spectral data acquired on-line and furthermore is assumed to add to the variation in the baseline shape. The main spectral absorption bands found in the fermentation samples occurred in the region $1000-1200 \mathrm{~cm}^{-1}$ and $1350-1450 \mathrm{~cm}^{-1}$. This is in alignment with the pure component spectra. The spectral fingerprint between $1000-1200 \mathrm{~cm}^{-1}$ represents the components glucose, glycerol, ethanol and phosphates. Note that, the spectral fingerprint of glucose 'shines through', dominating this lower spectral region and spectral variation due to glycerol, ethanol, and phosphates being present as fine differences in the 
dominating glucose fingerprint, was not observable to the naked eye. The upper region $1350-1450 \mathrm{~cm}^{-1}$ represents the analytes ammonium and acetate.

As expected, absorption changes due to the presence of peptides (amide III absorption bands) and yeast cells described in the region between 1200 and $1350 \mathrm{~cm}^{-1}$ (Sakhamuri et al. 2001; Singh 2009) are not pronounced in the spectrum. The insignificance of yeast cells to the spectral absorption was furthermore confirmed by the direct comparison of spectra collected on cell-free and cell-containing samples of data set 4 (Supplementary Fig. S5) as well as by PCA analysis (Supplementary Fig. S6). In the PCA score plot spanned by the first and second principal component (Fig. S 6) cell-free and cell-containing samples of data set 4 and 5 overlay mostly, confirming that cells do not add to the spectral variance. However, in the spectra acquired on-line a peak of constant intensity appeared in the respective region between 1200 and $1350 \mathrm{~cm}^{-}$(Fig. 4 b). It is assumed that this peak might be explained by peptide hydrolysis and consumption and simultaneous cell growth, consequently not changing in its intensity.

\section{PLS model calibration and validation}

The six PLS models were calibrated based on data sets 1 and 6 combining standard process data and the synthetic samples to provide a calibration set in which the different components were to a large extent uncorrelated. The validation was performed on data set 2-5. In particular, the validation on data set 2 and 3 presents the direct comparison of the modeling of spectral data acquired with both, the novel NLIR prototype and the standard FTIR instrument (see below section 'Comparison between the NLIR and the FTIR instrument'). The validation on data set 2 - 5 was used to demonstrate and discuss the general model performance. It must be emphasized that PLS model validation was performed on external data, i.e. the data sets used for validation were not included in the calibration set and belonged to a separated fermentation batch. Fig. 5 and Fig. 6 show the PLS calibration models for the six analytes of interest and their validation. In Fig. 5 (PLS models and validation for ethanol, glucose and acetate) and Fig. 6 (PLS models and validation for glycerol, ammonium and phosphates) the validation is presented in form of the model-predicted data over the fermentation course (diamonds) in direct comparison to the reference values measured (solid line). In addition, Table 3 summarizes the most common model performance parameters linked to the calibration and validation process. 

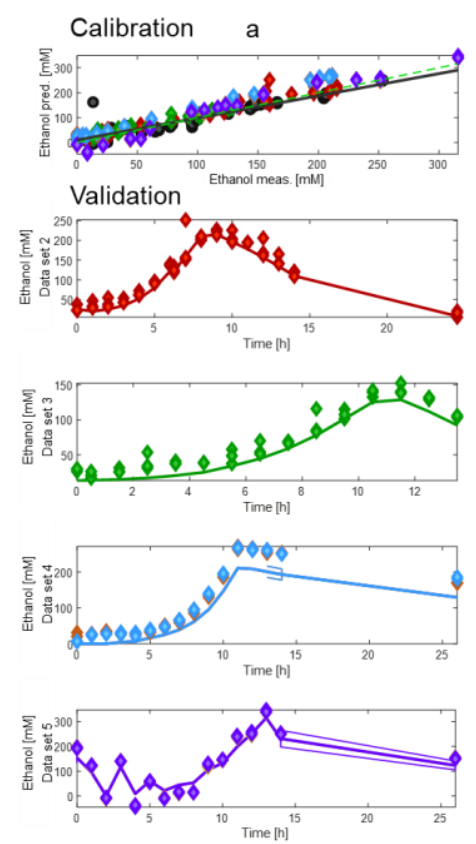

b
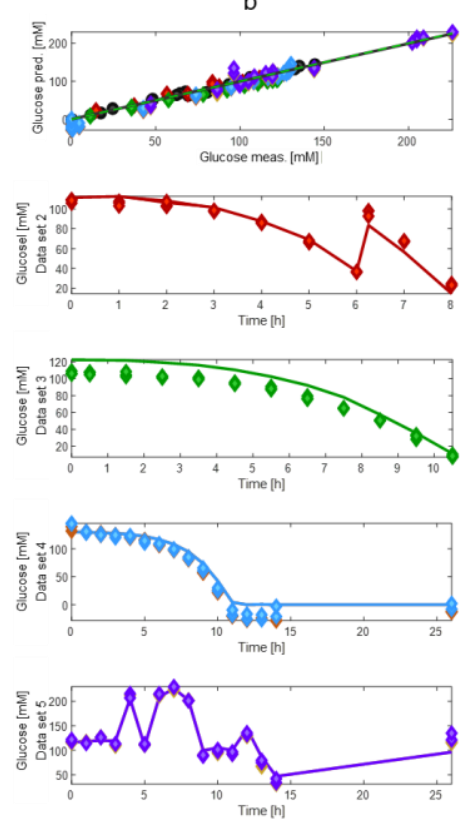
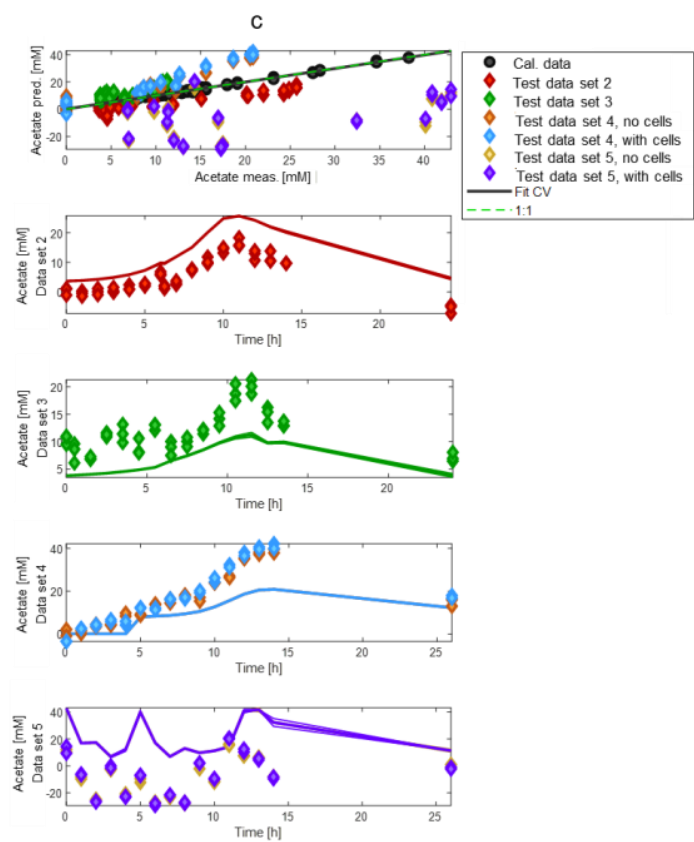

Fig. 5: PLS calibration models and model validation for a: Ethanol; b: Glucose and c: Acetate, with Cal. Data = Calibration data (data set $1+6$ ) and Fit $\mathrm{CV}$ as the fit for cross validated samples inside the calibration set. Model validation was performed on data sets 2-5 as indicated in the legend. For visualization, the reference data obtained by HPLC and assay analysis is presented as a solid line, and the predictions are presented as distinct data points. Note that, as the predictions, the reference data is not continuous but distinct. The borders around some reference values indicate a visible standard deviation (greater than $5 \%$ ) for the respective data point, or data point section, respectively
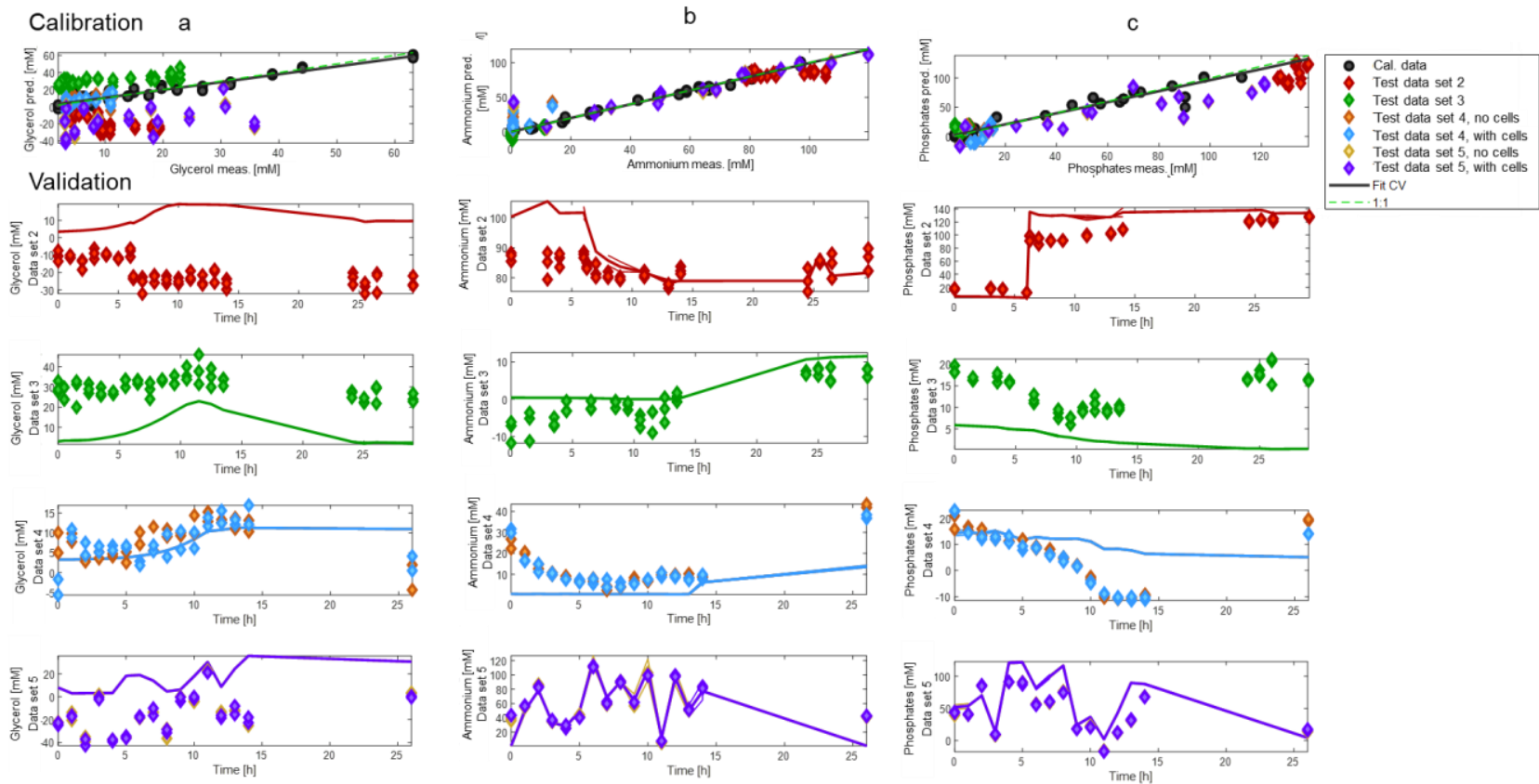

Fig. 6: PLS calibration model and model validation for a: Glycerol; b: Ammonium and c: Phosphates, with Cal. Data = Calibration data (data set $1+6$ ) and Fit $\mathrm{CV}$ as the fit for cross validated samples inside the calibration set. Model validation was performed on data sets 2-5 as indicated in the legend. For visualization, the reference data obtained by HPLC and assay 
analysis is presented as a solid line, and the predictions are presented as distinct data points. Note that, as the predictions, the reference data is not continuous but distinct. The borders around some reference values indicate a visible standard deviation (greater than $5 \%$ ) for the respective data point, or data point section, respectively

Table 3: Summary of PLS model parameters. The calibration was based on data set 1 and 6 and is represented with respect to the number of latent variables $(\mathrm{LV})$, the correlation coefficient between measured and predicted values $\mathbf{R}^{2}$, the RMSE (root-mean square error) and the bias between calibration and cross validation (CV). The validation for the data sets 2 - 5 is presented in terms of the relative root-mean-square error of prediction (RMSEP), the correlation coefficient between measured and predicted values in the validation set $R^{2}$ (Val) and bias

\begin{tabular}{|c|c|c|c|c|c|c|}
\hline $\begin{array}{l}\text { Model } \\
\text { parameter }\end{array}$ & Glucose & Glycerol & Acetate & Ethanol & Ammonium & Phosphates \\
\hline \multicolumn{7}{|c|}{ Calibration } \\
\hline Nr. of $L V$ & 5 & 9 & 14 & 5 & 9 & 3 \\
\hline $\begin{array}{l}\mathbf{R}^{2} \\
(\mathrm{Cal} / \mathrm{CV})\end{array}$ & 0.99 / 0.99 & $0.92 / 0.65$ & 0.99 / 0.94 & $0.89 / 0.82$ & $0.99 / 0.98$ & $0.95 / 0.92$ \\
\hline $\begin{array}{l}\text { RMSE [\%] } \\
(\mathrm{Cal} / \mathrm{CV})\end{array}$ & $2.8 / 3.3$ & $6.7 / 13.8$ & $2.1 / 5.9$ & $8.5 / 10.8$ & $2.6 / 4.7$ & $7.1 / 9.1$ \\
\hline $\begin{array}{l}\text { Bias [\%] } \\
(\mathrm{Cal} / \mathrm{CV})\end{array}$ & $-0.1 / 0.1$ & $0 /-0.4$ & $0 /-0.2$ & $0 / 0.4$ & $0 /-0.6$ & $0.1 /-0.3$ \\
\hline \multicolumn{7}{|c|}{ Validation per data set } \\
\hline \multicolumn{7}{|c|}{ Data set 2} \\
\hline RMSEP [\%] & 7.0 & 208.2 & 36.2 & 9.5 & 36.8 & 18.5 \\
\hline $\mathbf{R}^{2}($ Val $)$ & 0.96 & 0.39 & 0.9 & 0.95 & 0.3 & 0.94 \\
\hline Bias [\%] & 1.1 & -194.6 & -33.5 & 5.7 & -21.5 & -11.8 \\
\hline \multicolumn{7}{|c|}{ Data set 3} \\
\hline RMSEP [\%] & 12.3 & 102.2 & 74.0 & 13.7 & 43.2 & 209.8 \\
\hline $\mathbf{R}^{2}$ (Val) & 1.00 & 0.52 & 0.66 & 0.97 & 0.72 & 0.00 \\
\hline Bias [\%] & -11.4 & 99.2 & 67.5 & 12.1 & -34.8 & 193.3 \\
\hline \multicolumn{7}{|c|}{$\begin{array}{c}\text { Data set } 4 \\
(-/+)=(\text { cell-free } / \text { cell-containing })\end{array}$} \\
\hline $\begin{array}{l}\text { RMSEP [\%] } \\
(-/+)\end{array}$ & $12.2 / 9.9$ & $57.3 / 51.5$ & $45.7 / 52.4$ & $18.2 / 18.9$ & $95.3 / 92.7$ & 109.6 / 109.7 \\
\hline $\mathbf{R}^{2}$ (Val) & $0.99 / 0.98$ & $0.16 / 0.33$ & $0.92 / 0.94$ & $1.00 / 1.00$ & $0.56 / 0.44$ & $0.32 / 0.42$ \\
\hline Bias [\%] $(-/+)$ & $-9.5 /-6.6$ & $20.3 / 10.5$ & $36.6 / 41.4$ & $16.9 / 17.2$ & $77.8 / 75.2$ & $-60.4 /-67.3$ \\
\hline \multicolumn{7}{|c|}{$\begin{array}{c}\text { Data set } 5 \\
(-/+)=(\text { cell-free } / \text { cell-containing })\end{array}$} \\
\hline $\begin{array}{l}\text { RMSEP [\%] } \\
(-/+)\end{array}$ & $5.7 / 5.9$ & $99.2 / 102$ & $89.7 / 88$ & $8.8 / 9.2$ & $13.0 / 13.3$ & $23.1 / 22.7$ \\
\hline $\mathbf{R}^{2}$ (Val) & $0.97 / 0.96$ & $0.21 / 0.20$ & $0.20 / 0.17$ & $0.96 / 0.96$ & $0.84 / 0.84$ & $0.77 / 0.79$ \\
\hline $\operatorname{Bias}[\%](-/+)$ & $-1.6 / 0.1$ & $-88.2 /-91.4$ & $-80.3 /-77.2$ & $1.6 / 1.8$ & $3.3 / 3.6$ & $-16.7 /-16.5$ \\
\hline \multicolumn{7}{|c|}{$\begin{array}{c}\text { Average RMSEP [\%] } \\
\text { (column average, data set } 3 \text { and } 4 \text { were excluded in the case of phosphate and ammonium) }\end{array}$} \\
\hline & 8.8 & 103.4 & 63.6 & 13.1 & 21.0 & 21.4 \\
\hline \multicolumn{7}{|c|}{$\begin{array}{c}\mathbf{R}^{2} \text { (Val, all) } \\
\text { (calculated on data sets } 2-5 \text { as a whole) }\end{array}$} \\
\hline & 0.97 & 0.01 & 0.01 & 0.94 & 0.92 & 0.89 \\
\hline
\end{tabular}


With the exception of the model for ethanol, the calibration yielded an $\mathrm{R}^{2}$ greater than 0.95 , an RMSE of less than $10 \%$ and practically no bias (Table 3). The slightly worse calibration for ethanol is explained by one data point biasing the calibration fit, as observed in Fig. 5 a. The number of latent variables (LV) upon which the models were built suggested a good model robustness for phosphates $(3 \mathrm{LV})$, glucose and ethanol (5 LV each). The models for glycerol, ammonium and acetate were built upon 9, 9 and $14 \mathrm{LV}$, respectively, which is a rather high number, suggesting that these models are less robust and likely dependent on covariance structures, i.e. indirect predictions. For glycerol, the $\mathrm{R}^{2}$ of cross validation $(\mathrm{CV})$ is considerably decreased, also hinting towards predictions that are generally challenged for this component. Indirect predictions will be investigated in detail in the below section 'Investigation of indirect predictions'.

As emphasized visually in Fig. 5 and 6, the models for glucose and ethanol match the process data best with an RMSEP of on average $9 \%$ and $13 \%$, respectively (Table 3). The two models predicted reliably both, the process dynamics and the spiked samples, especially the latter demonstrating their robustness. The slightly worse predictions for ethanol compared to the predictions of glucose with respect to the reference data (Fig. 5) might be explained by the relatively low molecular absorption of ethanol (Fig. 3) making it more susceptible to spectral noise in the complex fermentation matrix. However, both components showed the overall best and equally good correlations between the measured and predicted concentration values $\left(\mathrm{R}^{2}\right.$ (Val) values, Table 3), which ranged between 0.94 and 1.00 in the different validation sets. In case of ammonium and phosphates, the validation yielded an RMSEP of on average $21 \%$ for both components, disregarding the validation on data sets 3 and 4 (as both components were present in low concentrations of less than $14 \mathrm{mM}$, Fig. 1). The models gave a good estimate of the process dynamics and corresponded well to spiking. However, the predictions showed a clear bias for some data points, in particular for predictions in data set 3 and 4 (Fig. $6 \mathrm{~b}$ and c). This bias from the measured concentration values is furthermore reflected in the decreased $\mathrm{R}^{2}(\mathrm{Val})$ values for these data sets (below 0.72 , Table 3). Nevertheless, the overall $\mathrm{R}^{2}$ (Val, all) value, calculated on the total number of samples of data sets $2-5$, resulted in a fairly good correlation between measured and predicted concentrations $\left(\mathrm{R}^{2}(\mathrm{Val}\right.$, all) of ca. 0.9). Previous work (Pontius et al. 2019), presenting PLS calibration models of ammonium and phosphates at comparable conditions (MIR spectra of synthetic, uncorrelated samples in a complex, however different, medium, calibration range $10-100 \mathrm{mM}$, external validation) showed considerably lower prediction errors of less than $5 \%$. The higher prediction errors obtained in this study despite the goodness of the calibration performance (Table 3 ) can be explained by an increased sample complexity (four more components were considered in the synthetic samples here, and besides, the validation was performed on fermentation samples, while only synthetic samples were considered in the previous work), striking the necessity to build calibration models under the relevant process conditions, considering 
the relevant species consumed and produced during the fermentation. Acetate and glycerol were predicted poorly with on average an RMSEP of $64 \%$ and $103 \%$, and an $\mathrm{R}^{2}$ (Val, all) value of only 0.01 . Both models did not correspond to spiked samples, indicating their lack of robustness, as do the number of LVs on which the models were built, and in the case of glycerol, the distinct decrease of $\mathrm{R}^{2}$ when comparing the results of calibration and cross validation.

In accordance with the literature (Bogomolov et al. 2015), and with the Supplementary Fig.s S5 and S6 showing the direct comparison of cell-free and cell-containing samples and the PCA score plot of all data sets, respectively, the presence of cells did not affect the spectra significantly and hence the prediction results. This is also confirmed in Fig. 5 and 6, presenting the validation on the data sets 4 and 5, and providing a direct comparison of the prediction of cell-free and cell-containing samples. In Fig. 5 and 6, the predictions of cell-free and cell-containing samples lie essentially on top of each other, as do the scores on the first and second principal component, for cell-free and cell-containing samples in Supplementary Fig. S6. However, a small, yet clear, off-set between the predictions of cell-free and cell-containing samples becomes obvious in the case of acetate and glycerol. This, once more, indicated that their prediction was based on covariance to a spectral region not corresponding to their actual absorption bands.

Notwithstanding the generally good performances in calibration for all components, the predictions for ammonium, phosphates, and in particular, glycerol and acetate were found to be challenged. Note that the different model performances in calibration and prediction became evident as the RMSEP was calculated based on external data sets. The gap between calibration performance and actual model performance strikes the need for external validation as performed in this work, to obtain a realistic idea of the models' applicability. As challenged predictions often boil down to indirect correlations within the data, i.e. indirect predictions, this was elaborated in detail in the below section.

\section{Investigation of indirect predictions}

As a general observation, the modeling of acetate and glycerol resulted in the poorest predictions across the validation sets. The following five considerations point out, that, within the process studied here, these two components were difficult to model, and their modeling was likely to rely on indirect predictions:

1) Both components were present in rather small concentrations (only up to $25 \mathrm{mM}$, Fig. 1).

2) Both components show a very low molecular absorption compared to the other species (Fig. 3).

3) Process correlations of both components were to a large extent decoupled by the synthetic (uncorrelated) samples. However, both components show overlapping spectral features with other components (glycerol with glucose, ethanol, and phosphates; acetate with ammonium (Fig. 3)). 
4) Models for both components responded poorly to spiking (Fig. 5 c and 6 a) and, in case of glycerol, resulted in a significant decrease in $\mathrm{R}^{2}(\mathrm{CV})$ compared to $\mathrm{R}^{2}(\mathrm{Cal})$.

5) The prediction for both components was slightly affected by the presence of cells (Fig.s 5 c and 6 a), described to cause variations at $1239 \mathrm{~cm}^{-1}$ and around $1540 \mathrm{~cm}^{-1}$ (Sivakesava et al. 2001). As both components show minor variations in the same region, the predictions will be biased if the absorbance in these regions is affected and this spectral variance is not included in the calibration data.

In order to investigate the assumption that acetate and glycerol were predicted indirectly, the VIP scores as a function of the wavenumber was studied in detail for each component, and this is presented as a heat map in Fig. 7.

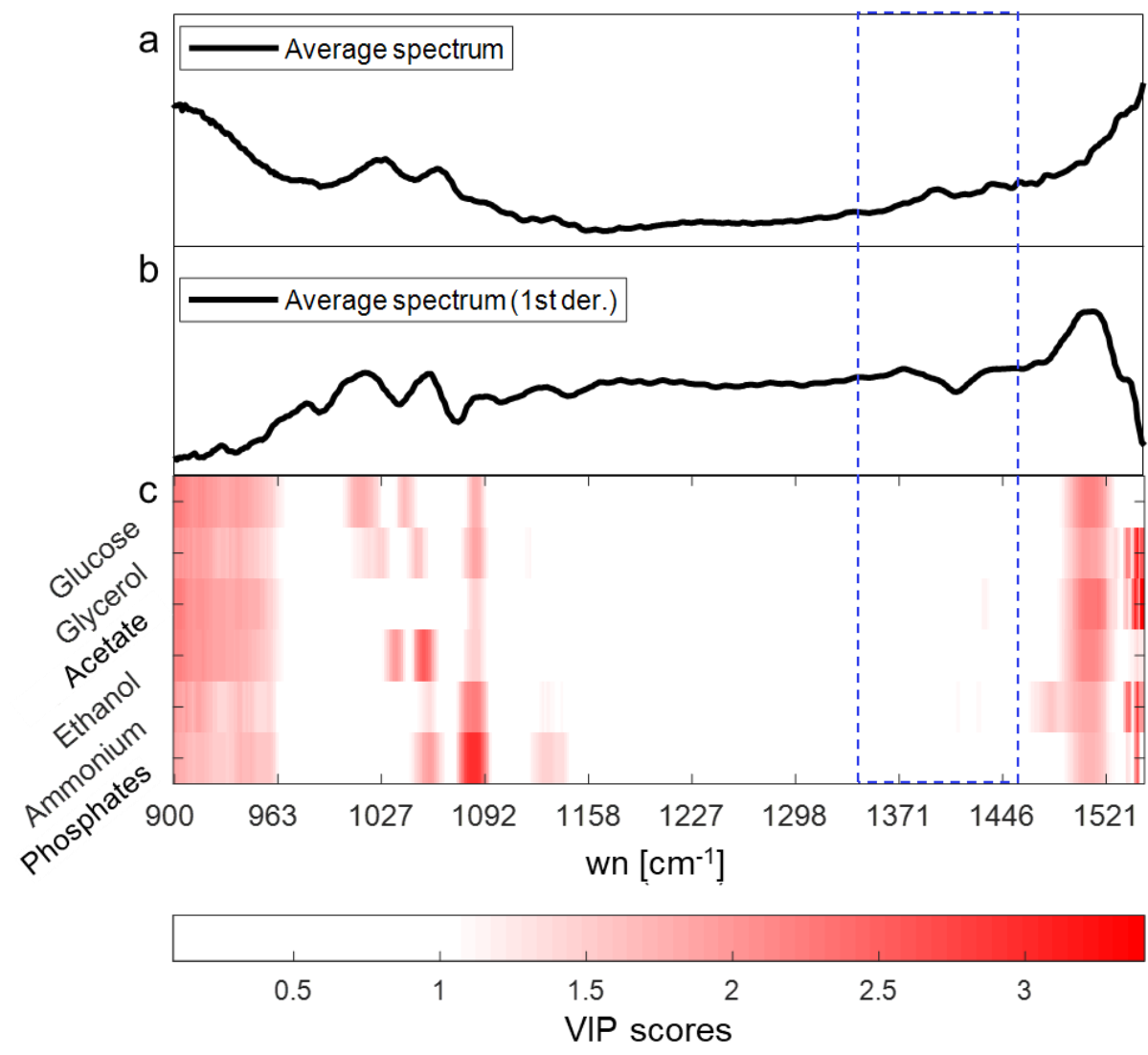

Fig. 7: a: Average spectrum of the calibration set (data set 1 and 6). b: Average spectrum of the calibration set preprocessed by mean-centering and the Savitzky-Golay algorithm (1st derivative and smoothing). c: Heat map of the VIP scores for the six PLS models for glucose, glycerol, acetate, ethanol, ammonium and phosphates. VIP scores greater than 1 generally indicate a high relevance of the respective variables (spectral region) for the PLS model

VIP scores greater than 1 generally indicate a high relevance of the respective variables (spectral region) for the PLS model. As can clearly be seen in Fig. 7, the VIP scores are highly overlapping. This can be expected according to the 
highly overlapping spectral features of the analytes of interest. However, it is important to notice that the region between 1350-1450 $\mathrm{cm}^{-1}$ (marked with a dashed line insert in Fig. 7) was not important at all for any of the models, although the major spectral peak for the components acetate and ammonium is located there (Fig. 3). Contrarily, the spectral borders were considered important for prediction, although only minor variation linked to the analytes occurred there. Summarizing, the inspection of the VIP scores revealed a valid correlation between the spectral fingerprint and the prediction of glucose, ethanol, glycerol and phosphates; however, it showed that the prediction of acetate and ammonium was based on a spectral region not corresponding to their actual absorption peaks. It can be concluded that all components were essentially modeled based on the same spectral regions implying that unique regression vectors for all six analytes were difficult to find. Besides, the VIP scores proposed that a variable selection might be beneficial to the models. In such a case, Fig. 7 clearly advocates that the variables in the region $960-1100 \mathrm{~cm}^{-1}$ and $1150-$ $1446 \mathrm{~cm}^{-1}$ do not contribute to the models, and thus might be excluded for the better. However, as previously shown by Pontius et. al (2019), a variable selection implying a decrease in variables for prediction can compromise the model performance when those variables contribute to the model stabilization. A brief check was performed investigating how a variable selection excluding the variables in the range $1158-1466 \mathrm{~cm}^{-1}$ affected the six models within this work. This variable selection resulted in essentially the same calibration performance for all six components. However, the predictions performed ambiguously. While the prediction performance remained essentially unchanged in case of the models for glucose, ethanol and phosphates (note that these components were built upon a low number of latent variables suggesting a fairly good robustness), it yielded contradictory results in case of the models for glycerol, acetate and ammonium. In the latter case, the performed variable selection indeed improved the prediction results in some validation sets, however, diminished the prediction results in most cases. The lack of robustness of these three models was previously assumed based on the high number of LV upon which these models were built, furthermore suggesting indirect covariance structures leading to indirect predictions.

As described by Berhe et al. (2016), two analytes will be predicted by the same linear combination of the predictor data, if the predictions of these two analytes are correlated, regardless their correlation in the raw data. In other words, also in uncorrelated samples as used for calibration in this work, the concentration prediction of two components might be correlated, even if the concentrations of these two components are not correlated in the calibration set. According to the procedure described by Berhe et al. (2016), all six analytes were investigated with respect to the occurrence of indirect covariance structures to one another, i.e. indirect predictions. The study of indirect predictions is presented in Fig. 8. 


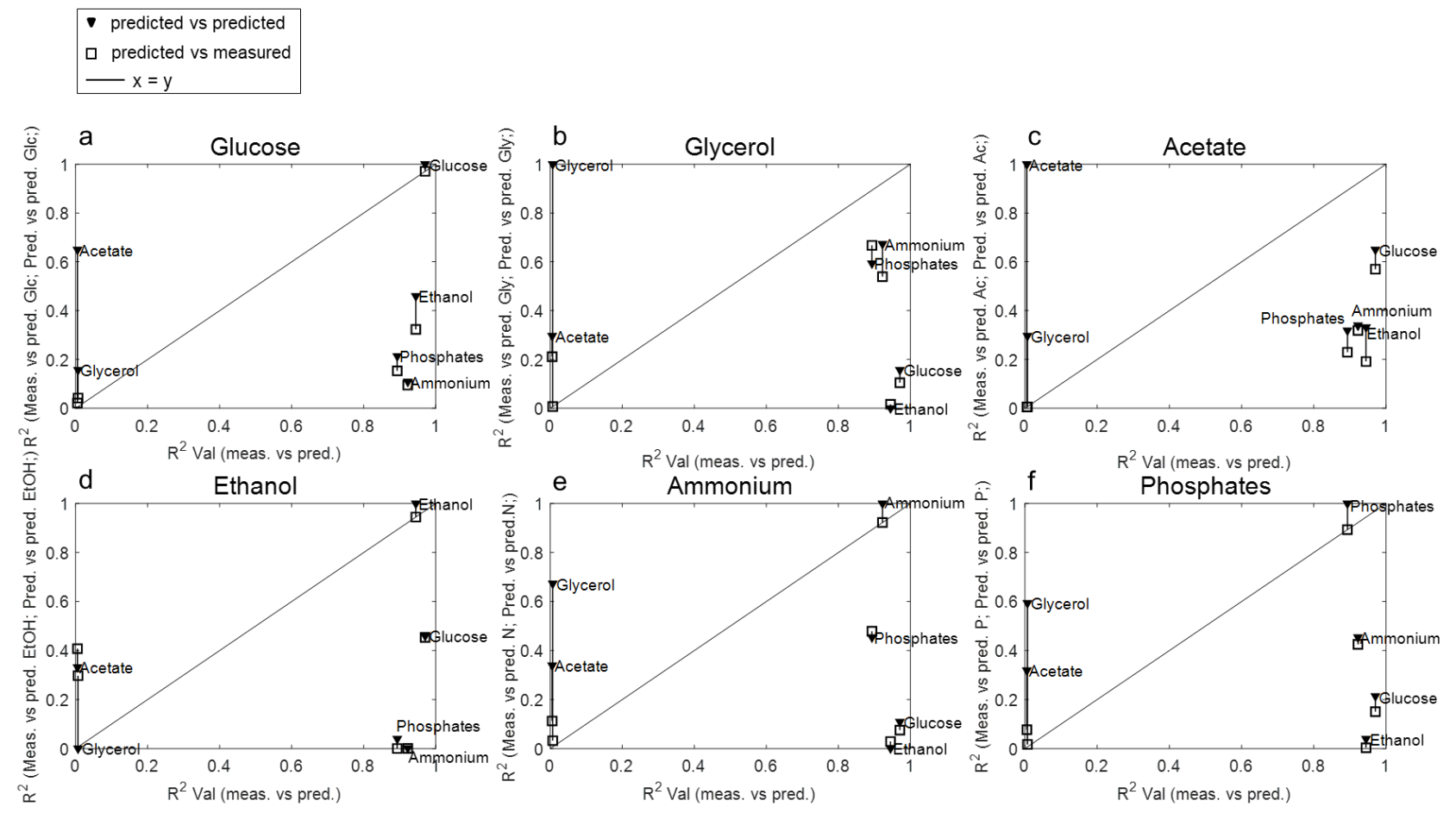

Fig. 8: Investigation of indirect predictions based on the prediction of a: Glucose (Glc), b: Glycerol (Gly), c: Acetate (Ac), d: Ethanol (EtOH), e: Ammonium (N) and f: Phosphates (P). The y-axis, exemplarily explained for Fig. 8 a), represents the correlation between the predicted analyte concentration and the measured glucose concentration (open square, 'predicted vs measured') and, respectively, between the predicted analyte concentration and the predicted glucose concentration (filled triangle, 'predicted vs predicted'). The $x$-axis represents the model performance expressed as the correlation coefficient $\left(R^{2}\right.$ Val) between measured and predicted analyte. The calculations as described by Berhe et al. (2016) were based on the total number o samples comprising the validation sets $2-5$.

The y-axis, exemplarily explained for Fig. 8 a, represents the correlation between the predicted analyte concentration, and the measured glucose concentration (open square, 'predicted vs. measured') and, respectively, the predicted analyte concentration and the predicted glucose concentration (filled triangle, 'predicted vs. predicted'). The x-axis represents the model performance expressed as the coefficient of determination $\left(\mathrm{R}^{2}\right)$ between measured and predicted analytes. The Fig. 8 b - f are to be read accordingly. Indirect predictions are indicated by a correlation between two analytes that is higher for 'predicted vs predicted' than for 'predicted vs measured'. Practically speaking, to spot an indirect prediction in Fig. 8, the filled triangle needs to show a significantly higher value on the y-axis than the corresponding open square, to which it is connected by a solid line. First of all, Fig. 8 was inspected to investigate if the prediction of glycerol, yielding the overall poorest predictions, was based on the prediction of one or several of the other components (Fig. 8 a, c - f). It could be concluded that the prediction of glycerol was essentially based on the prediction of both 
ammonium and phosphates (Fig. 8 e and f). To a smaller extent, the prediction of glycerol was found to be correlated with the prediction of acetate and glucose. Comparing these findings with the pure component spectra (Fig. 3), the correlation of glycerol with phosphates and glucose can be expected due to overlapping absorption bands in the region $1000-1150 \mathrm{~cm}^{-1}$. Contrarily, a correlation between glycerol and ammonium, explaining the most part of the glycerol prediction (highest correlation coefficient on the y-axis compared to phosphates, acetate and glucose) as well as the lesser correlation between glycerol and acetate is rather surprising and cannot be explained by spectral covariance. However, it fits with the observation in Fig. 7, that acetate and ammonium show overlapping VIP scores with glucose and phosphates around $1000 \mathrm{~cm}^{-1}$. Secondly, the prediction of acetate was investigated (Fig. 8 a, b, d - f). It was found that acetate was substantially explained by the prediction of glucose and to a lesser extent equally by the prediction of ammonium, phosphates, and glycerol. The correlation between the prediction of acetate and the prediction of glucose, phosphates and glycerol, once more, cannot be explained by spectral covariance but by overlapping VIP-scores. Contrarily, a correlation between the prediction of acetate with the prediction of ammonium is coherent with the pure component spectra exhibiting overlapping spectral features in the region $1400-1500 \mathrm{~cm}^{-1}$ (Fig. 3). However, as emphasized previously when inspecting the VIP scores, no high VIP scores were found in this region exhibiting the essential fingerprint of acetate and ammonium. Contrarily, according to Fig. 7 c, the prediction of ammonium and acetate is essentially based on the spectral borders and the region $1000-1150 \mathrm{~cm}^{-1}$, where only marginally pronounced absorption can be found. Furthermore, according to Fig. 8 b and c, glycerol and acetate are to the same extent predicted by respectively the other component. This explains the covariance found between glycerol and ammonium, or respectively, the correlation between acetate, glucose, glycerol and phosphates: Glycerol is to a certain extent explained by the prediction of acetate, which in turn, is explained by the prediction of mainly glucose and somewhat ammonium and phosphates. Vice versa, it can be observed that the prediction of ammonium relies to a small extent on the prediction of glycerol (Fig. 8 b) and the prediction of phosphates relies to a small extent on the prediction of acetate and glucose (Fig. 8 c). Thirdly, it can be observed that the prediction of glucose and ethanol are substantially independent from the prediction of any other component studied.

It can be concluded that within the current study, glucose and ethanol can be considered as predicted independently while the predictions of glycerol and acetate were trapped in a complex cage of covariance with each other and hence, with the predictions of glucose ammonium and phosphates. Thus, glycerol and acetate were not predicted independently but their predictions were to a large extent based on the predictions of ammonium and phosphates, and, respectively, glucose. While the prediction of glucose was based on the actual spectral fingerprint and hence a true correlation was 
valid for the prediction of glucose, ammonium was predicted based on VIP-scores not corresponding to the actual spectral fingerprint, and hence indirectly. The observation that the prediction of glycerol is to a large extent explained by the prediction of ammonium, which in turn, is predicted indirectly itself, explains why the prediction of glycerol was subject to the overall poorest prediction within this study. The predictions of ammonium and phosphates, vice versa, were found to be somewhat related to the predictions of glycerol and acetate, however to a much lesser extent.

Finally, it is important to mention that the extracted covariance structures were observed to be susceptible to the data used for validation, and the batch-to-batch variability occurring between the different data sets affected in the degree and nature of the complex covariance structures found. In other words, the nature and degree of the observed crosscorrelations were dependent on the validation data, however, the essential cage of covariance between those components remained. It is to bear in mind that such indirect models are only useful when a highly reproducible process is in place and their applicability will be limited when subject to a high batch-to-batch variability.

\section{Comparison between the NLIR and the FTIR instrument}

In order to compare the spectral quality achieved with the NLIR and the standard FTIR instrument, the model built on data set 1 and 6 was validated on data sets 2 and 3 as for these two data sets, spectra were acquired with both instruments. As the model was built equally on both spectral data sets, the RMSEP could be interpreted as a measure of spectral quality. The results are presented in Table 4.

Table 4: RMSEP values for the six models, built on spectral data collected with the NLIR and the FT-IR instrument, as distinguished in the table. For both spectral data sets, the models were validated on data set 2 and 3 (as a whole). The average RMSEP value was calculated based on the row values presented, disregarding glycerol and acetate as those values were based on indirect predictions

\begin{tabular}{l|rrrcrc}
\hline RMSEP & Glucose & Glycerol & Acetate & Ethanol & Ammonium & Phosphates \\
\hline FT-IR & $3.4 \%$ & $56.9 \%$ & $19.1 \%$ & $5.2 \%$ & $12.5 \%$ & $13.1 \%$ \\
average & \multicolumn{7}{c}{$8.6 \%$} \\
\hline NLIR & $10 \%$ & $133.9 \%$ & $43.1 \%$ & $8.7 \%$ & $7.4 \%$ & $14 \%$ \\
average & & & \multicolumn{2}{c}{$10.0 \%$} & \\
\hline
\end{tabular}


As demonstrated in Table 4, the prediction error is overall lower for the data acquired with the standard FTIR instrument, except for ammonium. Disregarding the models built for glycerol and acetate as found to be generally challenged by indirect predictions, on average, a model error of $8.6 \%$ and $10 \%$ was achieved with the standard FTIR instrument and the NLIR instrument, respectively. This indicates a higher sensitivity or, respectively, a better quality of the spectral data acquired with the FTIR instrument. The difference in spectral quality is evident in the spectra themselves, as demonstrated in Supplementary Fig. S7. However, the slight downgrading in spectral quality of NLIR spectra can be regarded as insignificant when used for PLS modeling. Generally, with both instruments, models yielded an error of less than $15 \%$ (excluding glycerol and acetate from the inspection), which is a reasonably good error with respect to industrial scale concentration predictions. It is worth mentioning that the change in spectral quality is not linear over the spectrum. The spectral quality is affected to different extents over different parts of the spectrum, as indicated by the approximately 3.5 percentage points larger RMSEP of the models built on NLIR data for glucose, ethanol and phosphates. However, other regions benefitted from the change in quality, as observed when modeling ammonium. The prediction error for this component improved by 5 percentage points when built on NLIR-data.

\section{On-line data acquisition}

Ultimately aiming at on-line monitoring of the components under study, the NLIR instrument was equipped with an ATR crystal designed as a flow-through-cell to facilitate on-line measurements. As phosphates and ammonium were not particularly relevant to the yeast fermentation process under study, since not added to the fermentation medium, their modeling was neglected. The spectral evolution of the flow-through data set is shown in Fig. 4 b. Due to the application of flow and a different ATR unit as such, the spectra collected on-line differed significantly from the spectra collected off-line as confirmed by the PCA score plot in Supplementary Fig. S5. Hence, the model could not be calibrated with the data collected off-line in data sets 1-6.

However, in order to demonstrate the potential of the on-line set-up, new models were built on the on-line spectra which were supplemented with HPLC data. In the following, these models will be called the 'flow-through' models. Subsequently, the flow-through models were applied to the spectra collected in between the reference points. The calibration and validation of the flow-through models is presented in Fig. 9. Additionally, the parameters relevant to the calibration are summarized in Table 5. 

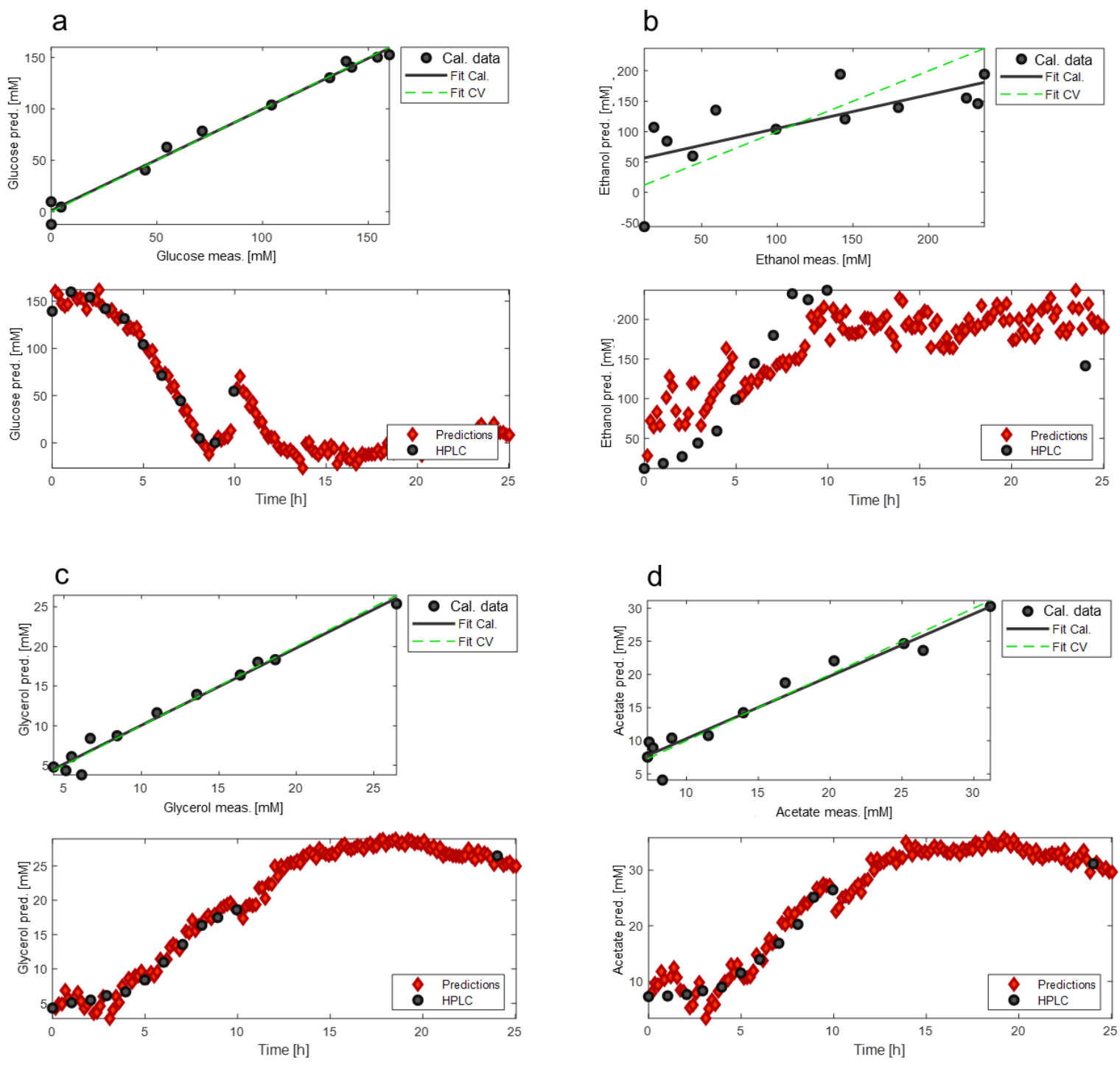

Fig. 9: Flow-through-model for a: Glucose, b: Ethanol, c: Glycerol and d: Acetate. The upper panel per subfigure shows the PLS calibration model, while the lower panel shows the prediction performance (internal validation)

Table 5: Calibration parameters for the flow-through model of the four analytes of interest

\begin{tabular}{|c|c|c|c|c|}
\hline Parameter & Glucose & Glycerol & Acetate & Ethanol \\
\hline \multicolumn{5}{|c|}{$\begin{array}{l}\text { Calibration } \\
12 \text { spectra collected on-line complemented with HPLC data }\end{array}$} \\
\hline Nr. of $L V$ & 3 & 5 & 4 & 2 \\
\hline $\mathbf{R}^{2}(\mathrm{Cal} / \mathrm{CV})$ & $0.99 / 0.95$ & $0.98 / 0.63$ & $0.94 / 0.57$ & $0.51 / 0.84$ \\
\hline $\begin{array}{l}\text { RMSE [\%] } \\
(\mathrm{Cal} / \mathrm{CV})\end{array}$ & $4 / 9.7$ & $4.4 / 19.3$ & $8 / 23.3$ & $26 / 15.6$ \\
\hline $\begin{array}{l}\text { Bias [\%] } \\
\text { (Cal / CV) }\end{array}$ & $0 /-1.5$ & $0 /-5.5$ & $0 /-7.3$ & $-1.3 / 4$ \\
\hline
\end{tabular}


As shown in Fig. 9 and reported in Table 5, the calibration of the flow-through models resulted in high $\mathrm{R}^{2}$ values of 0.94, 0.98 and 0.99, respectively, for acetate, glycerol and glucose. These values were in line with a small bias (max. $7.3 \%$ for acetate) and a small RMSE (Cal) (max. $8 \%$ for acetate). Note that, the components glycerol and acetate were predicted accurately in this data set (Fig. 9), as process correlations were not decoupled in the calibration data. The worst calibration was obtained for ethanol yielding an $\mathrm{R}^{2}$ (Cal) of 0.51 , complemented with an RMSE (Cal) of $26 \%$, and, nevertheless, a small bias of $-1.3 \%$. Cross-validation yielded certainly lower $\mathrm{R}^{2}$ values than the calibration, in particular for glycerol and acetate. In both cases, the $\mathrm{R}^{2}(\mathrm{CV})$ is around 0.6 resulting in a RMSE (CV) of around $20 \%$. The models for glucose and ethanol yielded better values for cross-validation $\left(\mathrm{R}^{2}(\mathrm{CV})\right), 0.95$ and 0.84 , respectively, reflecting a RMSECV of $10 \%$ and $15 \%$, respectively. All models were built on a significantly lower number of latent variables compared to the models built on the off-line ATR data, maximal 5 for glycerol, and then 4, 3, and 2 for modeling acetate, glucose and ethanol, respectively.

As advocated by the calibration parameters, the predictions performed fairly well, in particular for the components glucose, glycerol and acetate, while the prediction of ethanol was a bit more challenged. In case of the three former analytes, the spectra collected on-line every $10 \mathrm{~min}$ yielded a continuous trajectory resulting in a reliable component profile which is in very good agreement with the reference data points. Besides, the spiking of glucose after $9 \mathrm{~h}$, simultaneously diluting the fermentation broth and hence the other components, was captured accurately by the three models. It resulted in a peak and drop of prediction, respectively, in the glucose and glycerol / acetate profiles. It is worth to emphasize that the fermentation profile between $10 \mathrm{~h}$ and $24 \mathrm{~h}$ could be recovered without a single reference measurement. The resulting curve looks reasonable and is in agreement with the expected metabolism. The results are very promising regarding future predictions. The more biased predictions of ethanol obtained in this approach compared to the predictions yielded previously from off-line data might be explained by the variables used for prediction being particularly noisy. (Note that, using the NLIR instrument, noise does not spread linear over the spectrum and might have affected in particular the prediction of ethanol).

Notably, the flow-through ATR connected to the NLIR instrument was used in this work for the very first time. The approach demonstrated that fast and efficient spectral sampling and PLS modeling can be obtained by on-line spectra acquisition. 


\section{Discussion}

The application of IR-spectroscopy combined with PLS-modeling has been reported in several works to show immense potential to overcome the lack of monitoring strategies within the fermentation area, offering the possibility for multicomponent prediction (Dahlbacka et al. 2012; Fayolle et al. 2000; Genkawa et al. 2012; Koch et al. 2014; Kornmann et al. 2004; Landgrebe et al. 2010; Lourenço et al. 2012; Pollard et al. 2001; Sakhamuri et al. 2001). The versatility of this combined strategy has been reported for various processes covering mammalian cell culture, antibiotic fermentation with filamentous fungi, lactic acid fermentation, wine fermentation and, as studied in this work, yeast fermentation processes (dos Santos et al. 2017; Roychoudhury et al. 2006; Sakhamuri et al. 2001; Sandor et al. 2013; Sivakesava et al. 2001). However, generally little in-depth interpretation of developed calibration models with respect to spectral interpretation and correlation among variables has been done. Hence, overoptimistic results have often been reported considering the RMSEP yielded from internal validation as only performance statement. Within this work, the yeast performance parameters glucose, ethanol, glycerol and acetate, and additionally, ammonium and phosphates were modeled successfully, and importantly, applying novel NLIR technology. Due to their broad relevance in various fermentations, these parameters have been modeled by PLS based on IR data previously, mostly with similar performance results and limitations (Bogomolov et al. 2015; Diewok et al. 2004; Kornmann et. al. 2003; Roychoudhury et al. 2006; Sakhamuri et al. 2001; Sandor et al. 2013; Schenk et al. 2007; Sivakesava et al. 2001; Wu et al. 2015) as described in this work. Summarizing, in both, within the mentioned literature and the current study, the low concentration analytes were prone to being modeled based on covariance structures in the data sets rather than absorption bands directly associated with the individual components. Besides, batch-to-batch variability, low molecular absorption, and complex fermentation media definitely challenged the chemometric modelling, the latter implying that non-calibrated but appearing intermediates might bias the predictions during the dynamic fermentation process (Kornmann et.al. 2003). However, the modeling of six components within one fermentation case study has rarely been described and to our knowledge, the component matrix presented here has not been reported in such combination. Besides, this work demonstrated that within this set-up, in particular the modeling of glycerol and acetate was challenged by low concentration levels within the process and low IR activity compared to the other components under study, resulting in indirect predictions even though uncorrelated samples were used within the calibration set. Thus, PLS calibration models for glycerol and acetate were investigated in depth and shown to rely on indirect prediction, trapped in a cage of covariance with the prediction of ammonium, phosphates and glucose, or respectively, regions used 
for their prediction. Consequently, a high model error of $30 \%-200 \%$ was observed for both components in uncorrelated samples. Monitoring of glycerol and acetate by mid-IR spectroscopy during a yeast fermentation process was also found to be challenging by Schenk and coworkers (Schenk et al. 2007). Their work reports comparable high prediction errors of $50 \%-200 \%$ in uncorrelated samples and suggests a lower detection limit of $0.5 \mathrm{~g} / \mathrm{L}$ for each component (corresponding to $5 \mathrm{mM}$ glycerol and $8 \mathrm{mM}$ acetate, respectively). A considerable number of samples below this suggested detection limit was present in the data sets employed in this work and surely added to their challenged predictions. However, indirect predictions due to highly overlapping spectral features were not taken into account in the mentioned work. Such models, relying on process correlations can still be relevant and useful, if consistent and reproducible process conditions are provided. This applies especially to industrial production where processes must be operated under optimized and defined conditions (Kornmann et.al. 2003). However, in such a case, special attention must be given to the calibration maintenance of the model, as the indirect relationships will change with the process condition (e.g. medium, strain, feeding strategy, abundance of the analyte and other species). It is to bear in mind that models obtained for one process might not be transferred to another process due to a different process matrix. In other words, modeling the same component in different processes might indeed yield fairly different results.

The modification of the NLIR prototype as a flow-through unit enabled frequent and automated spectra collection, decreasing labor intensity while increasing data density. The first models applied to such on-line data were very promising, yielding representative analyte trajectories between $10 \mathrm{~h}$ and $24 \mathrm{~h}$, during which no reference data was obtained in the fermentation process. However, a new calibration procedure must be considered, since the spectra (baseline shape) acquired on-line differed significantly from the spectra acquired off-line due to the different ATR elements in use and the application of flow. Although the spectra acquired with the NLIR technology showed a slightly downgraded data quality compared to the standard FTIR instrument, as do previously described prototypes (Bogomolov et al. 2015), models resulted in comparable predictability (compared to the standard FTIR) yielding RMSEP values of less than $15 \%$ (excluding the models built for glycerol and acetate). Hence, the novel NLIR technology presented in this work can be seen as a valid alternative to the FTIR technology, besides being more suitable for an industrial production environment. Moreover, it was shown that when the natural process correlations were maintained in the calibration data, as was the case for the data collected on-line, the models of glycerol and acetate yielded concentration profiles in agreement with the reference data. These models relying on process correlations performed better than models built on uncorrelated samples, however, special attention must be given to their validity. Generally, it has been agreed on that, the quality of the calibration data has an essential impact on the model performance and robustness, and 
that direct models are most likely obtained with uncorrelated calibration samples. To this end, Bogomolov (2017) suggested recently a diagonal design for multi-component calibration problems, which may help to overcome the challenge of customized but possibly non-optimal calibration sample design dominating the literature. Moreover, combining real and synthetic samples inside the calibration set was previously found to deliver the best modeling results (Diewok et al. 2004), as did taking the first derivative during spectral preprocessing in comparable processes (Koch et al. 2014; Roychoudhury et al. 2006; Sakhamuri et al. 2001; Sivakesava et al. 2001). So far, novel technology opens the door to a more user-friendly application of spectroscopy instrumentation, yet, to turn the technology as such in an applicable soft sensor, expertise in chemometrics, process technology and process understanding are required. State-ofthe art chemometrics (mathematics) have been available for decades, however, general guidelines, methodologies and software standardizing and simplifying the modeling approach are still lacking and possibly the remaining bottleneck hindering a wider acceptance in industry. As recommended before by Luttmann et al. (2012), simplicity and standardization are a necessity for soft sensor application and increased implementation in industry. Consequently, in order to be outstanding, novel instrumentation should be complemented with proper software including all tools needed to turn the measurement method into an applicable soft sensor, generally simplifying the intense calibration procedure, and, importantly, providing guidance for multivariate calibration and model evaluation within the same interface.

\section{Author Contribution Statement}

KP conceived, designed and conducted the experimental research and analyzed experimental data. MB performed all chemometric modeling and related computational analysis. KP wrote the manuscript. All authors contributed to scientific discussions, reviewed and approved the final manuscript.

\section{Conflict of interest}

The authors declare that they have no conflict of interest.

\section{Ethical approval}

This article does not contain any studies with human participants or animals performed by any of the authors. 


\section{Acknowledgements}

We are grateful to the team of NLIR ApS (Farum, Denmark) for their technical support and providing useful information regarding their technology. Furthermore, we would like to acknowledge Frans van den Berg, University of Copenhagen, Department of Food Science, for providing the MatLab script for the bubble plots that are nicely visualizing the correlation patterns within the process. For providing the industrial relevant insulin precursor producing SIC strain, we kindly acknowledge Dina Petranovic Nielsen from Chalmers University, Biology and Biological Engineering. Finally, we wish to acknowledge the support of EU's Regional fund (BIOPRO-SMV project), Innovation Fund Denmark in the frame of the BIOPRO2 Strategic Research Center (grant agreement No. 4105-00020B), the European Union's Horizon 2020 research and innovation programme under the Marie Sklodowska-Curie grant agreement number 713683 (COFUNDfellowsDTU), the Danish Council for Independent Research (grant agreement No. 7017-00175A), and the Novo Nordisk Fonden in the frame of the Fermentation-Based Biomanufacturing education initiative. 


\section{References}

Barh, A., Pedersen, C., and Tidemand-Lichtenberg, P. (2017). Ultra-broadband mid-wave-IR upconversion detection. Opt. Lett., 42(8), 1504. https://doi.org/10.1364/ol.42.001504

Barnes, R. J., Dhanoa, M. S., and Lister, S. J. (1989). Standard Normal Variate Transformation and De-Trending of Near-Infrared Diffuse Reflectance Spectra. Appl. Spectrosc., 43(5), $772-777$. https://doi.org/10.1366/0003702894202201

Beebe, K. R., and Kowalski, B. R. (1987). An Introduction to Multivariate Calibration and Analysis. Anal. Chem., 59(17). https://doi.org/10.1021/ac00144a001

Berhe, D. T., Eskildsen, C. E., Lametsch, R., Hviid, M. S., van den Berg, F., and Engelsen, S. B. (2016). Prediction of total fatty acid parameters and individual fatty acids in pork backfat using Raman spectroscopy and chemometrics: Understanding the cage of covariance between highly correlated fat parameters. Meat Sci., 111, 18-26. https://doi.org/10.1016/j.meatsci.2015.08.009

Bogomolov, A., Heßling, M., Wenzel, U., Princz, S., Hellmuth, T., Bernal, M. J. B.; Sakharova, T., Usenov, I., Artyushenko, V., Meyer, H. (2015). Development and testing of mid-infrared sensors for in-line process monitoring in biotechnology. Sensors Actuators, B Chem., 221, 1601-1610. https://doi.org/10.1016/j.snb.2015.07.118

Buse, K., Kühnemann, F., Wolf, S., Kiessling, J., Kunz, M., and Popko, G. (2017). Upconversion-enabled array spectrometer for the mid-infrared, featuring kilohertz spectra acquisition rates. Opt. Express, $25(13), 14504$. https://doi.org/10.1364/oe.25.014504

Dahlbacka, J., Weegar, J., von Weymarn, N., and Fagervik, K. (2012). On-line measurement of the substrate concentrations in Pichia pastoris fermentations using FT-IR/ATR. Biotechnol. Lett., 34(6), $1009-1017$. https://doi.org/10.1007/s10529-012-0868-9

Dam, J. S., Høgstedt, L., Pedersen, C., Tidemand-Lichtenberg, P., and Andersen, H. V. (2016). Mid-infrared upconversion spectroscopy. J. Opt. Soc. Am. B, 33(11), D28. https://doi.org/10.1364/josab.33.000d28

Diewok, J., Baena, J. R., Mazarevica, G., Lendl, B., and Rosenberg, E. (2004). On-Line Fermentation Monitoring by Mid-Infrared Spectroscopy. Appl. Spectrosc., 58(7), 804-810. https://doi.org/10.1366/0003702041389229 
dos Santos, C. A. T., Páscoa, R. N. M. J., and Lopes, J. A. (2017). A review on the application of vibrational spectroscopy in the wine industry: From soil to bottle. TrAC - Trend. Anal. Chem., 88, 100-118. https://doi.org/10.1016/j.trac.2016.12.012

Eskildsen, C. E., Rasmussen, M. A., Engelsen, S. B., Larsen, L. B., Poulsen, N. A., and Skov, T. (2014). Quantification of individual fatty acids in bovine milk by infrared spectroscopy and chemometrics: Understanding predictions of highly collinear reference variables. J. Dairy Sci., 97(12), 7940-7951. https://doi.org/10.3168/jds.2014-8337

FDA (2004). Guidance for Industry - PAT A Framework for Innovative Pharmaceutical Development, Manufacturing, and Quality Assurance. (September), 1. https://doi.org/http://www.fda.gov/CDER/guidance/6419fnl.pdf

Fayolle, P., Picque, D., and Corrieu, G. (2000). On-line monitoring of fermentation processes by a new remote dispersive middle-infrared spectrometer. Food Control, 11(4), 291-296. https://doi.org/10.1016/S09567135(99)00105-X

Geladi, P., and Kowalski, B. R. (1986). Partial least-squares regression: a tutorial. Anal. Chim. Acta, 185(185), 1-17. https://doi.org/10.1016/0003-2670(86)80028-9

Genkawa, T., Watari, M., Nishii, T., and Ozaki, Y. (2012). Development of a Near-Infrared/Mid-Infrared Dual-Region Spectrometer for Online Process Analysis. Appl. Spectrosc., 66(7), 773-781. https://doi.org/10.1366/11-06499

Hermann, M., Pentek, T., and Otto, B. (2016). Design Principles for Industrie 4.0 Scenarios. 2016 49th Hawaii International Conference on System Sciences (HICSS), 3928-3937. https://doi.org/10.1109/HICSS.2016.488

Koch, C., Posch, A. E., Goicoechea, H. C., Herwig, C., and Lendl, B. (2014). Multi-analyte quantification in bioprocesses by Fourier-transform-infrared spectroscopy by partial least squares regression and multivariate curve resolution. Anal. Chim. Acta, 807, 103-110. https://doi.org/10.1016/j.aca.2013.10.042

Kornmann, H., Rhiel, M., Cannizzaro, C., Marison, I., and Von Stockar, U. (2003). Methodology for real-time, multianalyte monitoring of fermentations using an in-situ mid-infrared sensor. Biotechnol. Bioeng., 82(6), 702709. https://doi.org/10.1002/bit.10618

Kornmann, H., Valentinotti, S., Duboc, P., Marison, I., and von Stockar, U. (2004). Monitoring and control of Gluconacetobacter xylinus fed-batch cultures using in situ mid-IR spectroscopy. J. Biotechnol., 113(1-3), 231245. https://doi.org/10.1016/j.jbiotec.2004.03.029 
Landgrebe, D., Haake, C., Höpfner, T., Beutel, S., Hitzmann, B., Scheper, T., Rhiel, M., Reardon, K. F. (2010). On-line infrared spectroscopy for bioprocess monitoring. Appl. Microbiol. Biotechnol., 88(1), 11-22. https://doi.org/10.1007/s00253-010-2743-8

Liu, Z., Tyo, K. E. J., Martínez, J. L., Petranovic, D., and Nielsen, J. (2012). Different expression systems for production of recombinant proteins in Saccharomyces cerevisiae. Biotechnol. Bioeng. https://doi.org/10.1002/bit.24409

Lourenço, N. D., Lopes, J. A., Almeida, C. F., Sarraguça, M. C., and Pinheiro, H. M. (2012). Bioreactor monitoring with spectroscopy and chemometrics: a review. Anal. Bioanal. Chem., 404(4), $1211-1237$. https://doi.org/10.1007/s00216-012-6073-9

Luttmann, R., Bracewell, D. G., Cornelissen, G., Gernaey, K. V., Glassey, J., Hass, V. C., Kaiser, C., Preusse, C., Striedner, G., and Mandenius, C.-F. (2012). Soft sensors in bioprocessing: A status report and recommendations. Biotechnol. J., 7(8), 1040-1048. https://doi.org/10.1002/biot.201100506

Miller, C. E. (2000). Chemometrics for on-line spectroscopy applications - theory and practice. J. Chemom., 14(5-6), 513-528. https://doi.org/10.1002/1099-128X(200009/12)14:5/6<513::AID-CEM626>3.0.CO;2-5

Pavia, D. L., Lampman, G. M., and Kriz, G. S. (2001). Introduction to spectroscopy: a guide for students of organic chemistry. Retrieved from https://findit.dtu.dk/en/catalog/2304903416

Pearson, K. (1901). LIII. On lines and planes of closest fit to systems of points in space. London, Edinburgh, Dublin Philos. Mag. J. Sci., 2(11), 559-572. https://doi.org/10.1080/14786440109462720

Larkin, P. (2011). Infrared and Raman Spectroscopy: Principles and Spectral Interpretation. Infrared Raman Spectrosc. Princ. Spectr. Interpret., Elsevier Inc., pp. 73-115, https://doi.org/10.1016/c2010-0-68479-3.

Petersen, N., Ödman, P., Padrell, A. E. C., Stocks, S., Lantz, A. E., and Gernaey, K. V. (2010). In situ near infrared spectroscopy for analyte-specific monitoring of glucose and ammonium in streptomyces coelicolor fermentations. Biotechnol. Prog., 26(1), 263-271. https://doi.org/10.1002/btpr.288

Pollard, D. J., Buccino, R., Connors, N. C., Kirschner, T. F., Olewinski, R. C., Saini, K., Salmon, P. M. (2001). Realtime analyte monitoring of a fungal fermentation, at pilot scale, using in situ mid-infrared spectroscopy. Bioprocess Biosyst. Eng., 24(1), 13-24. https://doi.org/10.1007/s004490100226 
Pontius, K., Praticò, G., Larsen, F. H., Skov, T., Arneborg, N., Lantz, A. E., and Bevilacqua, M. (2019). Fast measurement of phosphates and ammonium in fermentation-like media: a feasibility study. N. Biotechnol. https://doi.org/10.1016/j.nbt.2019.11.006

Rinnan, Å., Berg, F. van den, and Engelsen, S. B. (2009). Review of the most common pre-processing techniques for near-infrared spectra. TrAC Trends Anal. Chem., 28(10), 1201-1222. https://doi.org/10.1016/j.trac.2009.07.007

Roychoudhury, P., Harvey, L. M., and McNeil, B. (2006). At-line monitoring of ammonium, glucose, methyl oleate and biomass in a complex antibiotic fermentation process using attenuated total reflectance-mid-infrared (ATR-MIR) spectroscopy. Anal. Chim. Acta, 561(1-2), 218-224. https://doi.org/10.1016/j.aca.2006.01.037

Sakhamuri, S., Bober, J., Irudayaraj, J., and Demirci, A. (2001). Simultaneous determination of multiple components in lactic acid fermentation using FT-MIR, NIR and FT-raman spectroscopic techniques. Process Biochem., 37, 371378.

Sandor, M., Rüdinger, F., Bienert, R., Grimm, C., Solle, D., and Scheper, T. (2013). Comparative study of non-invasive monitoring via infrared spectroscopy for mammalian cell cultivations. J. Biotechnol., 168(4), 636-645. https://doi.org/10.1016/j.jbiotec.2013.08.002

Savitzky, A., and Golay, M. J. E. (1964). Smoothing and Differentiation of Data by Simplified Least Squares Procedures. Anal. Chem., 36(8), 1627-1639. https://doi.org/10.1021/ac60214a047

Schenk, J., Marison, I. W., and von Stockar, U. (2007). Simplified Fourier-transform mid-infrared spectroscopy calibration based on a spectra library for the on-line monitoring of bioprocesses. Anal. Chim. Acta, 591(1 SPEC. ISS.), 132-140. https://doi.org/10.1016/j.aca.2007.03.056

Singh, B. R. (2009). Basic Aspects of the Technique and Applications of Infrared Spectroscopy of Peptides and Proteins. 2-37. https://doi.org/10.1021/bk-2000-0750.ch001

Sivakesava, S., Irudayaraj, J., and Demirci, A. (2001). Monitoring a bioprocess for ethanol production using FT-MIR and FT-Raman spectroscopy. J. Ind. Microbiol. Biotechnol., 26(4), $185-190$. https://doi.org/10.1038/sj.jim.7000124

Sjöström, M., Wold, S., Lindberg, W., Persson, J.-Å., and Martens, H. (1983). A multivariate calibration problem in analytical chemistry solved by partial least-squares models in latent variables. Anal. Chim. Acta, 150(150), 61- 
70. https://doi.org/10.1016/S0003-2670(00)85460-4

Skou, P. B., Berg, T. A., Aunsbjerg, S. D., Thaysen, D., Rasmussen, M. A., and van den Berg, F. (2017). Monitoring Process Water Quality Using Near Infrared Spectroscopy and Partial Least Squares Regression with Prediction Uncertainty Estimation. Appl. Spectrosc., 71(3), 410-421. https://doi.org/10.1177/0003702816654165

Sonnleitner, B., and Käppeli, O. (1986). Growth ofSaccharomyces cerevisiae is controlled by its limited respiratory capacity: Formulation and verification of a hypothesis. Biotechnol. Bioeng., 28(6), 927-937. https://doi.org/10.1002/bit.260280620

Steele, D. (1990). Multivariate calibration. Spectrochim. Acta Part A Mol. Spectrosc., 46(10), 1541. https://doi.org/10.1016/0584-8539(90)80069-B

Subramanian, A., and Rodriguez-Saona, L. (2009). Fourier Transform Infrared (FTIR) Spectroscopy. In Infrared Spectroscopy for Food Quality Analysis and Control (pp. 145-178). https://doi.org/10.1016/B978-0-12-374136$3.00007-9$

Sun, D. W. (2009). Infrared Spectroscopy for Food Quality Analysis and Control. Infrared Spectrosc. Food Qual. Anal. Control. https://doi.org/10.1016/B978-0-12-374136-3.X0001-6

von Schalien, R., Fagervik, K., Saxén, B., Ringbom, K., and Rydström, M. (1995). Adaptive on-line model for aerobic Saccharomyces cerevisiae fermentation. Biotechnol. Bioeng., $\quad 48(6), \quad 631-638$. https://doi.org/10.1002/bit.260480611

Westad, F., Bevilacqua, M., Marini, F. (2013). Regression. Data Handl. Sci. Technol., $28,127-170$. https://doi.org/10.1016/B978-0-444-59528-7.00004-1

Westad, F., and Marini, F. (2015). Validation of chemometric models - A tutorial. Anal. Chim. Acta, 893, $14-24$. https://doi.org/10.1016/j.aca.2015.06.056

Wold, S., Esbensen, K., and Geladi, P. (1987). Principal component analysis. Chemom. Intell. Lab. Syst., 2(1-3), 3752. https://doi.org/10.1016/0169-7439(87)80084-9

Wold, S., Johansson, E., Cocchi, M. (1993) PLS- Partial Least Squares Projections to Latent Structures in Drug Design: Theory, Methods and Applications, Escom Science Publishers, Leiden, The Netherlands, pp. 523-550. 
Wu, H., Brorson, K., Chavez, B., White, M., Khan, M., Read, E., \& Agarabi, C. (2015). Real time monitoring of bioreactor mAb IgG3 cell culture process dynamics via Fourier transform infrared spectroscopy: Implications for enabling cell culture process analytical technology. Front. Chem. Sci. Eng., 9(3), 386-406. https://doi.org/10.1007/s11705-015-1533-3 